\title{
Thermal Equilibria of Magnetically Supported, Black Hole Accretion Disks
}

\author{
H. Oda ${ }^{1}$, M. Machida ${ }^{2}$, K.E. Nakamura ${ }^{3}$ and R. Matsumoto ${ }^{4}$
}

\begin{abstract}
We present new thermal equilibrium solutions for optically thin and optically thick disks incorporating magnetic fields. The purpose of this paper is to explain the bright hard state and the bright/slow transition observed in the rising phases of outbursts in black hole candidates. On the basis of the results of three-dimensional MHD simulations, we assume that magnetic fields inside the disk are turbulent and dominated by the azimuthal component and that the azimuthally averaged Maxwell stress is proportional to the total (gas, radiation, and magnetic) pressure. We prescribe the magnetic flux advection rate to determine the azimuthal magnetic flux at a given radius. Local thermal equilibrium solutions are obtained by equating the heating, radiative cooling, and heat advection terms.

We find magnetically supported $\left(\beta=\left(p_{\text {gas }}+p_{\text {rad }}\right) / p_{\text {mag }}<1\right)$, thermally stable solutions for both optically thin disks and optically thick disks, in which the heating enhanced by the strong magnetic field balances the radiative cooling. The temperature in a low- $\beta$ disk $\left(T \sim 10^{7-11} \mathrm{~K}\right)$ is lower than that in an advection dominated accretion flow (or radiatively inefficient accretion flow) but higher than that in a standard disk. We also study the radial dependence of the thermal equilibrium solutions.

The optically thin, low- $\beta$ branch extends to $\dot{M} \gtrsim 0.1 \dot{M}_{\text {Edd }}$ where $\dot{M}$ is the mass accretion rate and $\dot{M}_{\text {Edd }}$ is the Eddington mass accretion rate, in which the temperature anti-correlates with the mass accretion rate. Thus optically thin low- $\beta$ disks can explain the bright hard state. Optically thick, low- $\beta$ disks have the radial dependence of the effective temperature $T_{\text {eff }} \propto \varpi^{-3 / 4}$. Such disks will be observed as staying in a high/soft state. Furthermore, limit cycle oscillations between an optically thick low- $\beta$ disk and a slim disk will occur because the optically thick low- $\beta$ branch intersects with the radiation pressure dominated standard disk branch. These limit cycle oscillations will show a smaller luminosity variation than that between a standard disk and a slim disk.
\end{abstract}

Subject headings: accretion, accretion disks — black hole physics - magnetic fields — X-ray: binaries

\footnotetext{
${ }^{1}$ Graduate School of Science and Technology, Chiba University, 1-33 Yayoi-cho, Inage-ku, Chiba 263-8522, Japan; oda@astro.s.chiba-u.ac.jp

${ }^{2}$ Division of Theoretical Astronomy, National Astronomical Observatory of Japan, 2-21-1 Osawa, Mitaka, Tokyo 181-8588, Japan

${ }^{3}$ Department of Sciences, Matsue National College of Technology, 14-4 Nishiikuma-cho, Matsue, Shimane 6908515, Japan

${ }^{4}$ Department of Physics, Graduate School of Science, Chiba University, 1-33 Yayoi-cho, Inage-ku, Chiba 2638522, Japan
}

\section{Introduction}

X-ray spectral states of galactic black hole candidates (BHCs) have been classified on the basis of their spectral shape and X-ray luminosity. In the high/soft state, the X-ray spectrum is dominated by a modified blackbody (thermal) component with characteristic temperature $\sim 1 \mathrm{keV}$. The low/hard state is observed at low luminosity $\left(L<0.1 L_{\mathrm{Edd}}\right)$, where $L_{\mathrm{Edd}}=4 \pi c G M / \kappa_{\mathrm{es}} \sim$ $1.47 \times 10^{39}\left(M / 10 M_{\odot}\right)\left(\kappa_{\mathrm{es}} / 0.34 \mathrm{~cm}^{2} \mathrm{~g}^{-1}\right)^{-1} \mathrm{erg} \mathrm{s}^{-1}$ is the Eddington luminosity. Here $M$ is the black hole mass and $\kappa_{\mathrm{es}}$ is the electron scattering opac- 
ity. The X-ray spectrum in the low/hard state is dominated by a power-law component (photon index $\Gamma \sim 1.7$ ) with an exponential cutoff at $\sim 200 \mathrm{keV}$. The very high/steep power law state is observed at high luminosity $\left(\sim L_{\text {Edd }}\right)$, in which the blackbody component and the powerlaw component become comparable. Super critical accretion flows have been identified by some authors in narrow-line Seyfert 1 galaxies (NLS1s; e.g., Kawaguchi 2003) and in ultraluminous Xray sources (ULXs; e.g., Vierdavanti et al. 2006, 2008). The spectral fitting models used in their paper are based on the slim disk model (e.g., Abramowicz et al. 1988). These objects are referred to as staying in the slim disk state.

Recently, Mivakawa et al. (2008) analyzed the results of RXTE observations of the black hole candidate GX 339-4 in the rising phases of transient outbursts. When the luminosity is lower than $\sim 0.07 L_{\mathrm{Edd}}$, the X-ray spectrum is dominated by a power-law component with an exponential cutoff, and the cutoff energy is roughly constant $\sim 200 \mathrm{keV}$. This is a characteristic feature of the low/hard state. By contrast, when the luminosity becomes higher than $\sim 0.07 L_{\mathrm{Edd}}$, the cutoff energy anti-correlates with the luminosity and decreases to $\sim 50 \mathrm{keV}$. This suggests that the electron temperature of a disk decreases as the luminosity increases. Furthermore, this state has been observed up to $\sim 0.3 L_{\text {Edd. }}$. Miyakawa et al. (2008) have named it the "bright hard state".

Gierliński \& Newton (2006) have reported two types of hard-to-soft transitions. One is the bright/slow transition that occurs at $\sim 0.3 L_{\text {Edd }}$ and takes more than 30 days. The other is the dark/fast transition which occurs at less than $0.1 L_{\text {Edd }}$ and takes less than 15 days (see also Done \& Gierliński 2003).

In the conventional theory of accretion disks, the concept of phenomenological $\alpha$-viscosity is introduced. In this framework, the $\varpi \varphi$-component of the stress tensor is assumed to be proportional to the sum of the gas pressure $p_{\text {gas }}$ and radiation pressure $p_{\mathrm{rad}}, t_{\varpi \varphi}=\alpha_{\mathrm{SS}}\left(p_{\text {gas }}+p_{\mathrm{rad}}\right)$. Here $\alpha_{\mathrm{SS}}$ is the viscosity parameter introduced by Shakura \& Sunvaev (1973). The standard disk model was developed by Shakura \& Sunvaev (1973), under the assumption that a substantial fraction of the viscously dissipated energy in an optically thick disk is radiated locally. This stan- dard disk model reproduced the thermal component in the high/soft state but cannot explain the hard X-ray spectrum observed in the low/hard state.

Thorne \& Price (1975) proposed that hard Xrays from Cyg X-1 are produced in an inner optically thin hot disk. Shibazaki \& Hōshi (1975) studied the structure and stability of optically thin hot accretion disks. Eardley, Lightman, \& Shapiro (1975) and Shapiro, Lightman. \& Eardley (1976, hereafter SLE) constructed a model for optically thin two-temperature accretion disks in which the ion temperature is higher than the electron temperature. However, these disks are thermally unstable.

Ichimaru (1977) pointed out the importance of energy advection in hot, magnetized accretion flows, and obtained steady solutions of optically thin disks. In this model, the viscously dissipated energy is stored in the gas as entropy and advected into the central object rather than being radiated. Such geometrically thick, optically thin, advection-dominated accretion flows (ADAFs) or radiatively inefficient accretion flows (RIAFs) have been studied extensively by Naravan \& Yi (1994, 1995), and Abramowicz et al. (1995). Esin et al. (1997) found that the maximum luminosity of the ADAF/RIAF solutions is $0.4 \alpha^{2} L_{\text {Edd }}$. These solutions explain the low/hard state.

However, the ADAF/RIAF solutions cannot explain the high luminosity observed in the bright hard state and during the bright/slow transition unless $\alpha \sim 1$. Furthermore, the ADAF/RIAF solutions do not show an anti-correlation between the luminosity and the electron temperature (therefore, the cutoff energy) observed in the bright hard state.

In the light of this discussion, our main aim is to propose steady models of magnetically supported black hole accretion disks that can explain the bright hard state and the bright/slow transition.

Okada et al. (1989) constructed a model of non-relativistic equilibrium tori with purely azimuthal magnetic fields in the pseudo-Newtonian potential (Paczyńsky \& Wiita 1980), which has been used as initial conditions of numerical simulations (e.g., Machida, Havashi, \& Matsumoto 
2000; Machida \& Matsumoto 2003; Machida et al. 2006). Komissarov (2006) developed a model of relativistic equilibrium tori with strong toroidal magnetic fields around a rotating black hole. However, the angular momentum transport was not taken into account in their models.

The origin of the efficient angular momentum transport (that is, $\alpha$-viscosity) in accretion disks had been a puzzle until Balbus \& Hawley (1991) pointed out the importance of the magnetorotational instability (MRI). The MRI can excite and maintain magnetic turbulence. The Maxwell stress generated by turbulent magnetic fields dominates the total stress and efficiently transports the angular momentum of the disk material. Many researchers have studied the growth and saturation of the MRI by conducting local and global magnetohydrodynamic (MHD) simulations.

Global three-dimensional MHD simulations of optically thin, radiatively inefficient accretion disks indicate that the amplification of magnetic fields becomes saturated when $\beta=p_{\text {gas }} / p_{\text {mag }} \sim$ 10 , and that then the disk approaches a quasisteady state. In this quasi-steady state, the ratio of the azimuthally averaged Maxwell stress to the azimuthally averaged gas pressure, which corresponds to the $\alpha$-parameter in the conventional theory of accretion disks, is $\sim 0.01-0.1$ (e.g., Hawley 2000; Machida, Hayashi, \& Matsumoto 2000; Hawley \& Krolik 2001; Machida \& Matsumoto $2003)$.

Three-dimensional MHD simulations have revealed that magnetic fields contribute to not only the angular momentum transport but also to disk heating via the dissipation of magnetic energy inside the disk (e.g., Machida et al. 2006; Hirose et al. 2006; Krolik et al. 2007).

Machida et al. (2006) found that when the mass accretion rate exceeds the threshold for the onset of a cooling instability, an optically thin, radiatively inefficient, hot, gas pressure dominated accretion disk (ADAF/RIAF-like disk) evolves towards an optically thin, radiatively efficient, cool, magnetic pressure dominated accretion disk (low- $\beta$ disk). In their simulation, when the density of the accretion disk exceeds a critical density, the cooling instability taking place in the ADAF/RIAF-like disk results in a decrease in the gas pressure. Thus the disk shrinks in the vertical direction. During that time, the azimuthal magnetic flux $\left\langle B_{\varphi}\right\rangle H$ is almost conserved because the cooling time scale is faster than that of the buoyant escape of the magnetic flux, where $\left\langle B_{\varphi}\right\rangle$ is the mean azimuthal magnetic field, and $H$ is the half thickness of the disk. As the disk shrinks, the magnetic pressure exceeds the gas pressure because the magnetic pressure increases due to flux conservation. Then the disk is supported by magnetic pressure and stops shrinking. In such low- $\beta$ disks, the magnetic flux cannot escape through the Parker instability (Parker 1966) because the strong magnetic tension suppresses the growth of the Parker instability (see Shibata, Tajima, \& Matsumoto 1990). Therefore the disk stays in a quasi-equilibrium cool state.

In this state, the dissipated magnetic energy is mainly radiated (not advected), and the Maxwell stress is proportional to the sum of the gas pressure and the magnetic pressure. The luminosity of the disk becomes higher than that of the initial ADAF/RIAF state. These numerical results indicate that a luminous hard state exists in BHCs.

Oda et al. (2007) constructed a steady model of optically thin accretion disks on the basis of these results from three-dimensional MHD simulations of accretion disks. They found that when the mass accretion rate exceeds the threshold for the onset of thermal instability, the thermally and viscously stable low- $\beta$ branch appears. These solutions confirmed the results by Machida et al. (2006). On this branch, the luminosity emitted by an accretion disk can exceed $0.2 L_{\text {Edd }}$. Furthermore, the mean temperature is lower than that of the ADAF/RIAF (higher than that of the standard disk) and anti-correlates with the mass accretion rate. Therefore, they concluded that optically thin low- $\beta$ disks can explain the bright/hard state.

In this paper, we extend the model of optically thin low- $\beta$ disks to the optically thick regime. We obtain local thermal equilibrium curves of accretion disks incorporating the magnetic fields. The total pressure is the sum of the gas pressure, the radiation pressure, and the magnetic pressure. We consider the thermal bremsstrahlung radiation in the optically thin limit and blackbody radiation in the optically thick limit as radiative cooling. To complete the set of basic equations, we specify the advection rate of the azimuthal magnetic flux. The basic equations are presented in $\S 2$. In 
$\S 3$ we obtain the thermal equilibrium curves and study their radial dependence. $\S 4$ is devoted to a

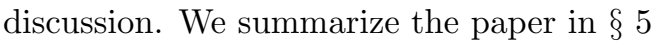

\section{Models and Assumptions}

\subsection{Basic Equations}

We extended the basic equations for onedimensional steady black hole accretion flows (e.g., Kato et al. 2008), incorporating magnetic fields. We adopt cylindrical coordinates $(\varpi, \varphi, z)$. General relativistic effects are simulated using the pseudo-Newtonian potential $\psi=-G M /\left(r-r_{\mathrm{s}}\right)$ (Paczyńsky \& Wiita 1980), where $G$ is the gravitational constant, $M$ is the black hole mass (we assume $M=10 M_{\odot}$ in this paper $), r=\left(\varpi^{2}+z^{2}\right)^{1 / 2}$, and $r_{\mathrm{s}}=2 G M / c^{2}$ is the Schwarzschild radius.

We start with the resistive MHD equations

$$
\begin{gathered}
\frac{\partial \rho}{\partial t}+\nabla \cdot(\rho \boldsymbol{v})=0 \\
\rho\left[\frac{\partial \boldsymbol{v}}{\partial t}+(\boldsymbol{v} \cdot \nabla) \boldsymbol{v}\right]= \\
-\rho \nabla \psi-\nabla\left(p_{\text {gas }}+p_{\mathrm{rad}}\right)+\frac{\boldsymbol{j} \times \boldsymbol{B}}{c}, \\
\frac{\partial(\rho \epsilon)}{\partial t}+\nabla \cdot\left[\left(\rho \epsilon+p_{\text {gas }}+p_{\mathrm{rad}}\right) \boldsymbol{v}\right] \\
-(\boldsymbol{v} \cdot \nabla)\left(p_{\mathrm{gas}}+p_{\mathrm{rad}}\right)=q^{+}-q^{-} \\
\frac{\partial \boldsymbol{B}}{\partial t}=\nabla \times\left(\boldsymbol{v} \times \boldsymbol{B}-\frac{4 \pi}{c} \eta \boldsymbol{j}\right)
\end{gathered}
$$

where $\rho$ is the density, $\boldsymbol{v}$ is the velocity, $\boldsymbol{B}$ is the magnetic field, $\boldsymbol{j}=c \nabla \times \boldsymbol{B} / 4 \pi$ is the current density, and $\rho \epsilon=p_{\text {gas }} /(\gamma-1)+3 p_{\text {rad }}$ is the internal energy. Here, $\gamma=5 / 3$ is the specific heat ratio and $p_{\text {gas }}=\mathcal{R} \rho T / \mu$ is the gas pressure where $\mathcal{R}$ is the gas constant, $\mu$ is the mean molecular weight (we assumed to be 0.617), and $T$ is the temperature. In the energy equation (3),$q^{+}$is the heating rate, and $q^{-}$is the radiative cooling rate. In the induction equation (4), $\eta \equiv c^{2} / 4 \pi \sigma_{\mathrm{c}}$ is the magnetic diffusivity, where $\sigma_{\mathrm{c}}$ is the electric conductivity.

\subsubsection{Azimuthally Averaged Equations}

Three-dimensional global MHD and local RadiationMHD simulations of black hole accretion disks showed that magnetic fields inside the disk are turbulent and dominated by the azimuthal component in a quasi-steady state (e.g., Machida et al. 2006; Hirose et al. 2006). On the basis of results of these simulations, we decomposed the magnetic fields into the mean fields $\overline{\boldsymbol{B}}=\left(0,\left\langle B_{\varphi}\right\rangle, 0\right)$ and fluctuating fields $\delta \boldsymbol{B}=\left(\delta B_{\varpi}, \delta B_{\varphi}, \delta B_{z}\right)$ and also decomposed the velocity into the mean velocity $\overline{\boldsymbol{v}}=\left(v_{\varpi}, v_{\varphi}, v_{z}\right)$ and the fluctuating velocity $\delta \boldsymbol{v}=\left(\delta v_{\varpi}, \delta v_{\varphi}, \delta v_{z}\right)$. Here \langle\rangle denotes the azimuthal average. We assume that the fluctuating components vanish when azimuthally averaged, $\langle\delta \boldsymbol{v}\rangle=\langle\delta \boldsymbol{B}\rangle=0$, and that the radial and vertical components of the magnetic fields are negligible compared with that of the azimuthal component, $\left|\left\langle B_{\varphi}\right\rangle+\delta B_{\varphi}\right| \gg\left|\delta B_{\varpi}\right|,\left|\delta B_{z}\right|$ (see panel (a) in figure 11).

Let us derive the azimuthally averaged equations. We assume that the disk is in a steady state and in hydrostatic balance in the vertical direction. By azimuthally averaging equations (1) - (4) and ignoring the second order terms of $\delta \boldsymbol{v}$, $\delta B_{\varpi}$, and $\delta B_{z}$, we obtain

$$
\begin{aligned}
& \frac{1}{\varpi} \frac{\partial}{\partial \varpi}\left(\varpi \rho v_{\varpi}\right)+\frac{\partial}{\partial z}\left(\rho v_{z}\right)=0, \\
& \rho v_{\varpi} \frac{\partial v_{\varpi}}{\partial \varpi}+\rho v_{z} \frac{\partial v_{\varpi}}{\partial z}-\frac{\rho v_{\varphi}^{2}}{\varpi} \\
& =-\rho \frac{\partial \psi}{\partial \varpi}-\frac{\partial p_{\mathrm{tot}}}{\partial \varpi}-\frac{\left\langle B_{\varphi}^{2}\right\rangle}{4 \pi \varpi}, \\
& \rho v_{\varpi} \frac{\partial v_{\varphi}}{\partial \varpi}+\rho v_{z} \frac{\partial v_{\varphi}}{\partial z}+\frac{\rho v_{\varpi} v_{\varphi}}{\varpi}= \\
& \frac{1}{\varpi^{2}} \frac{\partial}{\partial \varpi}\left[\varpi^{2} \frac{\left\langle B_{\varpi} B_{\varphi}\right\rangle}{4 \pi}\right]+\frac{\partial}{\partial z}\left(\frac{\left\langle B_{\varphi} B_{z}\right\rangle}{4 \pi}\right), \\
& 0=-\frac{\partial \psi}{\partial z}-\frac{1}{\rho} \frac{\partial p_{\text {tot }}}{\partial z}, \\
& \frac{\partial}{\partial \varpi}\left[\left(\rho \epsilon+p_{\text {gas }}+p_{\text {rad }}\right) v_{\varpi}\right]+\frac{v_{\varpi}}{\varpi}\left(\rho \epsilon+p_{\text {gas }}+p_{\text {rad }}\right) \\
& +\frac{\partial}{\partial z}\left[\left(\rho \epsilon+p_{\text {gas }}+p_{\text {rad }}\right) v_{z}\right]-v_{\varpi} \frac{\partial}{\partial \varpi}\left(p_{\text {gas }}+p_{\text {rad }}\right) \\
& -v_{z} \frac{\partial}{\partial z}\left(p_{\text {gas }}+p_{\text {rad }}\right)=q^{+}-q^{-} \\
& 0=-\frac{\partial}{\partial z}\left[v_{z}\left\langle B_{\varphi}\right\rangle\right]-\frac{\partial}{\partial \varpi}\left[v_{\varpi}\left\langle B_{\varphi}\right\rangle\right] \\
& +\{\nabla \times\langle\delta \boldsymbol{v} \times \delta \boldsymbol{B}\rangle\}_{\varphi}-\{\eta \nabla \times(\nabla \times \overline{\boldsymbol{B}})\}_{\varphi},
\end{aligned}
$$


where $p_{\text {tot }}=p_{\text {gas }}+p_{\text {rad }}+p_{\text {mag }}$ is the total pressure and $p_{\text {mag }}=\left\langle B_{\varphi}^{2}\right\rangle / 8 \pi$ is the azimuthally averaged magnetic pressure. The third and fourth terms on the right-hand side of equation (10) represent the dynamo term and the magnetic diffusion term which we approximate later on the basis of the results of the numerical simulations. Note that we have considered only the azimuthal component of the induction equation (4).

\subsubsection{Vertically Integrated, Azimuthally Aver- aged Equations}

We assume that the radial velocity $v_{\varpi}$, the specific angular momentum $\ell=\varpi v_{\varphi}$, and $\beta \equiv$ $\left(p_{\text {gas }}+p_{\text {rad }}\right) / p_{\text {mag }}$ are independent of $z$, and we assume a polytropic relation $p_{\text {tot }}=K \rho^{1+1 / N}$ in the vertical direction, where $N$ is the polytropic index (we adopt $N=3$ in this paper). Vertical integration of equation (8) yields

$$
\begin{gathered}
\rho(\varpi, z)=\rho_{0}(\varpi)\left(1-\frac{z^{2}}{H^{2}}\right)^{N}, \\
p_{\text {tot }}(\varpi, z)=p_{\text {tot0 }}(\varpi)\left(1-\frac{z^{2}}{H^{2}}\right)^{N+1},
\end{gathered}
$$

and we assume

$$
T(\varpi, z)=T_{0}(\varpi)\left(1-\frac{z^{2}}{H^{2}}\right),
$$

where $H$ is the half thickness of the disk given by

$$
\Omega_{\mathrm{K} 0}^{2} H^{2}=2(N+1) \frac{p_{\text {tot } 0}}{\rho_{0}},
$$

where $\Omega_{\mathrm{K} 0}=(G M / \varpi)^{1 / 2} /\left(\varpi-r_{\mathrm{s}}\right)$ is the Keplerian angular velocity. Here the subscript 0 refers to quantities in the equatorial plane. Under these assumptions, the surface density $\Sigma$ and the vertically integrated total pressure $W_{\text {tot }}$ are given by

$$
\begin{aligned}
\Sigma & \equiv \int_{-H}^{H} \rho d z \\
& =\int_{-H}^{H} \rho_{0}\left(1-\frac{z^{2}}{H^{2}}\right)^{N} d z \\
& =2 \rho_{0} I_{N} H, \\
W_{\mathrm{tot}} & \equiv \int_{-H}^{H} p_{\mathrm{tot}} d z
\end{aligned}
$$

$$
\begin{aligned}
& =\int_{-H}^{H} p_{\text {tot } 0}\left(1-\frac{z^{2}}{H^{2}}\right)^{N+1} d z \\
& =2 p_{\text {tot } 0} I_{N+1} H
\end{aligned}
$$

where $I_{N}=\left(2^{N} N !\right) /(2 N+1)$ !. Using $\Sigma$ and $W_{\text {tot }}$, equation (14) can be rewritten as

$$
\Omega_{\mathrm{K} 0}^{2} H^{2}=(2 N+3) \frac{W_{\text {tot }}}{\Sigma} .
$$

We note that the half thickness of the disk depends on not only the gas and radiation pressure but also on the magnetic pressure.

We now integrate the other basic equations in the vertical direction and assume Keplerian rotation $\left(\Omega=\Omega_{\mathrm{K} 0}\right)$ instead of equation ([6). We obtain

$$
\begin{gathered}
\dot{M}=-2 \pi \varpi \Sigma v_{\varpi} \\
\Omega=\Omega_{\mathrm{K} 0} \\
\dot{M}\left(\ell_{\mathrm{K} 0}-\ell_{\mathrm{in}}\right)=-2 \pi \varpi^{2} \int_{-H}^{H} \frac{\left\langle B_{\varpi} B_{\varphi}\right\rangle}{4 \pi} d z \\
\frac{\dot{M}}{2 \pi \varpi^{2}} \frac{W_{\text {gas }}+W_{\mathrm{rad}}}{\Sigma} \xi=Q^{+}-Q_{\mathrm{rad}}^{-} \\
\dot{\Phi} \equiv \int_{-H}^{H} v_{\varpi}\left\langle B_{\varphi}\right\rangle d z \\
=\int_{\varpi}^{\varpi_{\text {out }}} \int_{-H}^{H}\left[\{\nabla \times\langle\delta \boldsymbol{v} \times \delta \boldsymbol{B}\rangle\}_{\varphi}\right. \\
\left.-\{\eta \nabla \times(\nabla \times \overline{\boldsymbol{B}})\}_{\varphi}\right] d \varpi d z+\text { const. }(22)
\end{gathered}
$$

where $\dot{M}$ is the mass accretion rate, $\ell_{\mathrm{K} 0}=\varpi^{2} \Omega_{\mathrm{K} 0}$ is the Keplerian angular momentum and $\ell_{\text {in }}$ is the specific angular momentum swallowed by the black hole. $W_{\text {gas }}$ and $W_{\text {rad }}$ are the vertically integrated gas and radiation pressures, respectively. The left-hand side of the energy equation has been simplified by introducing the entropy gradient parameter $\xi$ (see Kato et al. 2008). On the righthand side, $Q^{+}$is the vertically integrated heating rate, and $Q_{\text {rad }}^{-}$is the vertically integrated radiative cooling rate. In equation (22), $\dot{\Phi}$ is the radial advection rate of the azimuthal magnetic flux (hereafter we call it the magnetic flux advection rate). 


\section{2. $\alpha$-prescription of the Maxwell Stress Tensor}

Global MHD simulations of radiatively inefficient, magnetic pressure supported (low- $\beta$ ) disks (Machida et al. 2006) showed that the ratio of the azimuthally averaged Maxwell stress to the sum of the azimuthally averaged gas pressure and magnetic pressure is nearly constant except in the plunging region $\left(\alpha_{\mathrm{B}} \equiv-\left\langle B_{\varpi} B_{\varphi} / 4 \pi\right\rangle /\left\langle p_{\text {gas }}+\right.\right.$ $\left.\left.p_{\text {mag }}\right\rangle \sim 0.05-0.1\right)$. Global MHD simulations of radiatively inefficient, gas pressure supported (high- $\beta$ ) disks also implied that $\alpha_{\mathrm{B}}$ is nearly constant because $\alpha_{\mathrm{m}} \equiv-\left\langle B_{\varpi} B_{\varphi} / 4 \pi\right\rangle /\left\langle p_{\mathrm{mag}}\right\rangle \sim 0.2-$ 0.5 and $\beta \sim 10$ are nearly constant inside the disk (e.g., Hawley \& Krolik 2001).

Following the simulation results, we assume that the azimuthally averaged $\varpi \varphi$-component of the Maxwell stress inside a disk is proportional to the total (gas, radiation, and magnetic) pressure

$$
\frac{\left\langle B_{\varpi} B_{\varphi}\right\rangle}{4 \pi}=-\alpha p_{\text {tot }} .
$$

Integrating in the vertical direction, we obtain

$$
\int_{-H}^{H} \frac{\left\langle B_{\varpi} B_{\varphi}\right\rangle}{4 \pi} d z=-\alpha W_{\mathrm{tot}} .
$$

This is one of the key assumptions in this paper. When the magnetic pressure is high, the stress level can be high even though the gas pressure and the radiation pressure are low.

\subsection{Heating and Cooling Rates}

\subsubsection{The Magnetic Heating Rate}

In the conventional theory, the viscous heating was expressed as $q_{\mathrm{vis}}^{+}=t_{\varpi \varphi} \varpi(d \Omega / d \varpi)$, where $t_{\varpi \varphi}$ is the $\varpi \varphi$-component of the total stress and $\Omega$ is the angular velocity, respectively. The results of three-dimensional MHD simulations indicate that dissipation due to the thermalization of magnetic energy dominates the total dissipative heating rate throughout a disk and is expressed as $q^{+} \sim\left\langle B_{\varpi} B_{\varphi} / 4 \pi\right\rangle \varpi(d \Omega / d \varpi)$ (e.g. Hirose et al. 2006; Machida et al. 2006; Krolik et al. 2007). Hereafter, we refer to it as the magnetic heating rate.

Following these simulation results, we employ magnetic heating as the heating mechanism inside a disk, and set the vertically integrated heating rate as follows:

$Q^{+}=\int_{-H}^{H}\left[\frac{\left\langle B_{\varpi} B_{\varphi}\right\rangle}{4 \pi} \varpi \frac{d \Omega}{d \varpi}\right] d z=-\alpha W_{\mathrm{tot}} \varpi \frac{d \Omega}{d \varpi}$,

where we have used equation (23). We note that if the magnetic pressure is high, the heating rate can also be large even when the gas pressure and the radiation pressure are low.

\subsubsection{The Radiative Cooling Rate}

We assume that radiative cooling is provided by the thermal bremsstrahlung emission in the optically thin limit. Thus the vertically integrated optically thin cooling rate is given by

$$
\begin{aligned}
Q_{\text {thin }}^{-} & =\int_{-H}^{H} 6.2 \times 10^{20} \rho^{2} T^{1 / 2} d z \\
& =6.2 \times 10^{20} \frac{I_{2 N+1 / 2}}{2 I_{N}{ }^{2}} \frac{\Sigma^{2}}{H} T_{0}{ }^{1 / 2} .
\end{aligned}
$$

In addition, we assume radiative cooling due to blackbody radiation in the optically thick limit. The vertically integrated optically thick cooling rate is expressed as

$$
Q_{\text {thick }}^{-}=\frac{16 \sigma I_{N} T_{0}^{4}}{3 \tau / 2},
$$

where $\sigma$ is the Stefan-Boltzmann constant, $\tau=$ $\tau_{\text {abs }}+\tau_{\text {es }}$ is the total optical depth, $\tau_{\text {abs }}$ is the absorption optical depth, $\tau_{\mathrm{es}}=0.5 \kappa_{\mathrm{es}} \Sigma$ is the electron scattering optical depth, and $\kappa_{\mathrm{es}}=$ $0.34 \mathrm{~cm}^{2} \mathrm{~g}^{-1}$ is the electron scattering opacity.

Although we should solve the transfer equation in the intermediate case, we use the following approximate form (e.g., Hubenv 1990; Narayan \& Yi 1995; Abramowicz et al. 1996),

$$
Q^{-}=\frac{16 \sigma I_{N} T_{0}{ }^{4}}{3 \tau / 2+\sqrt{3}+\tau_{\mathrm{abs}^{-1}}},
$$

where

$\tau_{\text {abs }}=\frac{Q_{\text {thin }}^{-}}{Q_{\text {thick }}^{-}}=\frac{6.2 \times 10^{20}}{16 \sigma} \frac{I_{2 N+1 / 2}}{2 I_{N}{ }^{3}} \frac{\Sigma^{3}}{H} T_{0}^{-7 / 2}$.

Using these approximations, the vertically integrated radiation pressure and the total pressure 
can be expressed as

$$
\begin{aligned}
W_{\text {rad }} & =\frac{Q^{-}}{4 c} \frac{I_{4}}{I_{N}} H\left[\frac{2}{3}\left(\frac{3 \tau}{2}+\sqrt{3}\right)\right], \\
W_{\text {tot }} & =W_{\text {gas }}+W_{\text {rad }}+W_{\text {mag }} \\
& =\left(1+\beta^{-1}\right)\left[\frac{I_{N+1}}{I_{N}} \frac{\mathcal{R}}{\mu} T_{0} \Sigma\right] \\
& +\left[\frac{Q^{-}}{4 c} \frac{I_{4}}{I_{N}} H\left[\frac{2}{3}\left(\frac{3 \tau}{2}+\sqrt{3}\right)\right]\right],
\end{aligned}
$$

respectively.

\subsection{Prescription of the Magnetic Flux Advection Rate}

We complete the set of basic equations by specifying the radial distribution of the mean azimuthal magnetic fields. If we ignore the dynamo term and the magnetic diffusion term and perform the integration in the left-hand side of the induction equation (22), we obtain

$$
\dot{\Phi}=-v_{\varpi} B_{0}(\varpi) 2 \frac{I_{(N+1) / 2}}{\sqrt{I_{N}}} H=\text { const. }
$$

where

$$
B_{0}(\varpi)=2 \pi^{1 / 2}\left(\frac{\mathcal{R}}{\mu} T_{0}\right)^{1 / 2} \Sigma^{1 / 2} H^{-1 / 2} \beta^{-1 / 2}
$$

is the mean azimuthal magnetic field in the equatorial plane. However, $\dot{\Phi}$ is not always conserved in the radial direction due to the presence of the dynamo term and the magnetic diffusion term. According to Machida et al. (2006), this equals $\dot{\Phi} \propto \varpi^{-1}$ in the quasi steady state. Following this result, we parametrize the dependence of $\dot{\Phi}$ on $\varpi$ by introducing a parameter, $\zeta$, as follows.

$$
\dot{\Phi}(\varpi ; \zeta, \dot{M}) \equiv \dot{\Phi}_{\text {out }}(\dot{M})\left(\frac{\varpi}{\varpi_{\text {out }}}\right)^{-\zeta},
$$

where $\dot{\Phi}_{\text {out }}$ is the magnetic flux advection rate at the outer boundary $\varpi=\varpi_{\text {out }}$. When $\zeta=0$, the magnetic flux is conserved in the radial direction, and when $\zeta>0$ (or $\zeta<0$ ), the magnetic flux increases (or decreases) with a decreasing radius (see panel (b) in figure 1). The azimuthal magnetic flux can increase inside a disk when the azimuthal flux of opposite polarity buoyantly escapes from the disk (e.g., Nishikori. Machida \& Matsumoto 2006)

Equation (34) is the second key assumption in this paper. Specifying the magnetic flux advection rate enables the magnetic pressure to increase when the disk temperature and the disk thickness decrease. By contrast, if we specified the plasma $\beta$ at each radius instead of the magnetic flux advection rate, the decrease in the temperature results in a decrease in magnetic pressure. This is inconsistent with the results of three-dimensional MHD simulations (e.g., Machida et al. 2006).

\section{Results}

\subsection{Local Thermal Equilibrium Curves}

We solved the above basic equations at $\varpi=$ $5 r_{\mathrm{s}}$ to obtain local thermal equilibrium curves. We also assume $\ell_{\text {in }}=\ell_{\mathrm{K}}\left(3 r_{\mathrm{s}}\right)$ in the $\varphi$ component of the momentum equation (20). Results of the global three-dimensional MHD simulations of optically thin black hole accretion flows (e.g., Machida et al. 2004) indicate that $\xi=1$ (see also Oda et al. 2007). This positivity of $\xi$ means that the heat advection becomes a cooling at a fixed radius. In this paper, we adopt $\xi=1$ in both the optically thin regime and the optically thick regime.

To determine the flux advection rate at a fixed radius, we need to specify $\dot{\Phi}_{\text {out }}$. Hence, we imposed the outer boundary condition that $T_{\text {out }}=$ $T_{\text {virial }}=\left(\mu c^{2} / 3 \mathcal{R}\right)\left(\varpi_{\text {out }} / r_{\mathrm{s}}\right)^{-1}$ and $\beta_{\text {out }}=10$ at $\varpi_{\text {out }}=1000 r_{\mathrm{s}}$. Under these conditions, we obtained $\dot{\Phi}_{\text {out }}$ by solving equations (20), (24), (31), and (34). For given $\dot{M}, \alpha, \zeta$, and $\varpi$, we obtained local thermal equilibrium solutions.

Figure 2 shows the thermal equilibrium curves plotted in the $\Sigma$ vs. $\dot{M} / \dot{M}_{\text {Edd }}, T_{0}, \beta, \tau_{\text {eff }}$ plane for $\alpha=0.05, \zeta=0.6$ (thick solid), 0.3 (dashed), 0 (dotted), and -1.8 (thin solid), respectively. Here $\dot{M}_{\mathrm{Edd}}=L_{\mathrm{Edd}} / \eta_{\mathrm{e}} c^{2}=4 \pi G M /\left(\eta_{\mathrm{e}} \kappa_{\mathrm{es}} c\right)$ is the Eddington mass accretion rate, $\eta_{\mathrm{e}}=0.1$ is the energy conversion efficiency, $\tau_{\text {eff }}=0.5 \sqrt{\kappa_{\text {es }} \kappa_{\mathrm{ff}}} \Sigma$ is the effective optical depth, and $\kappa_{\mathrm{ff}}=6.4 \times$ $10^{22} \rho_{0} T_{0}{ }^{-3.5} \mathrm{~cm}^{2} \mathrm{~g}^{-1}$ is the opacity of free-free absorption, respectively.

When $\zeta$ has a large negative value $(\zeta=-1.8)$, which means that the magnetic field strength at $\varpi=5 r_{\mathrm{s}}$ is negligible, the ADAF/RIAF and SLE 
branches appear in the optically thin regime, and the standard and slim disk branches appear in the optically thick regime as expected. (Strictly speaking, although the low- $\beta$ branches discussed below also appear in much lower temperature regions, we do not display them because we assumed fully ionized plasmas.)

For large values of $\zeta$, we obtained new branches in both the optically thin and the optically thick regime. We call them "low- $\beta$ " branches. On the low- $\beta$ branches, the dissipated magnetic energy is mainly radiated rather than advected. Therefore, the disk temperature and the gas pressure are lower than in the ADAF/RIAF (but higher than in the standard disk). In contrast, the magnetic pressure is high because the mean azimuthal magnetic field increases as $H$ decreases. The low- $\beta$ branches consist of an optically thin part and an optically thick part. We find that the optically thin low$\beta$ branches extend to $\dot{M} \gtrsim 0.1 \dot{M}_{\text {Edd }}$ corresponding to $L \gtrsim 0.1 L_{\mathrm{Edd}}$ when $\zeta \gtrsim 0.3$. We note that the critical mass accretion rate for the existence of ADAF/RIAF solutions $\dot{M}_{\mathrm{c}, \mathrm{A}}$ is $\sim 0.1 \dot{M}_{\mathrm{Edd}}$ and that of the gas pressure dominated standard disk solutions $\dot{M}_{\mathrm{c}, \mathrm{S}}$ is $\sim 0.05 \dot{M}_{\mathrm{Edd}}$. The slim disk solution appears when $\dot{M} \gtrsim 5 \dot{M}_{\text {Edd }}$.

It is worth noting that $\dot{M} \propto \Sigma$ and $T_{0} \propto \Sigma^{-2}$ in the optically thin low- $\beta$ branches, and that $\dot{M} \propto \Sigma$ and $T_{0} \propto \Sigma^{1 / 2}$ in the optically thick low- $\beta$ branches. These relations are derived as follows. First let us derive the dependence of $\dot{\Phi}_{\text {out }}(\dot{M})$ on $\dot{M}$. In the range of $\dot{M} \lesssim \dot{M}_{\text {Edd }}$ in which the low- $\beta$ branches appear, $W_{\text {gas }} \gg W_{\text {rad }}$ at $\varpi_{\text {out }}$. From equations (17), (18), (20), and (31), we find that $W_{\text {tot,out }} \propto \dot{M}, \Sigma_{\text {out }} \propto \dot{M}$, $H_{\text {out }} \sim$ constant, and $v_{\varpi, \text { out }} \sim$ constant. Therefore equation (33) yields $B_{0}\left(\varpi_{\text {out }}\right) \propto \dot{M}^{1 / 2}$ and equation (32) yields $\dot{\Phi}_{\text {out }} \propto \dot{M}^{1 / 2}$. Next, we derive the dependence of $\dot{M}$ on $\Sigma$. When the magnetic pressure dominates the total pressure, $W_{\text {tot }} \sim B_{0}{ }^{2} H \propto \dot{\Phi}^{2} /\left(v_{\varpi}{ }^{2} H\right) \propto \dot{M} /\left(v_{\varpi}{ }^{2} H\right)$. Since equations (18), (20), and (17) yield $v_{\varpi} \propto \dot{M} / \Sigma$, $W_{\text {tot }} \propto \dot{M}, H \propto\left(W_{\text {tot }} / \Sigma\right)^{1 / 2} \propto(\dot{M} / \Sigma)^{1 / 2}$, we find that $\dot{M} \propto \Sigma$ and $H$ is constant. Finally we derive the dependence of $T_{0}$ on $\Sigma$ by noting that magnetic heating $\left(Q^{+} \propto W_{\text {tot }} \propto \dot{M} \propto \Sigma\right)$ balances radiative cooling on the low- $\beta$ branches. We find from $Q^{-} \propto \Sigma^{2} T_{0}^{1 / 2}$ that $T_{0} \propto \Sigma^{-2}$ in the optically thin regime, whereas we find from $Q^{-} \propto T_{0}{ }^{4} / \Sigma$ that $T_{0} \propto \Sigma^{1 / 2}$ in the optically thick regime.
Figure 3 depicts thermal equilibrium curves for the same parameters as in figure 2 but plotted in the $T_{0}$ vs. $\dot{M} / \dot{M}_{\text {Edd }}$ plane and the $\tau_{\text {eff }}$ vs. $\dot{M} / \dot{M}_{\text {Edd }}$ plane. On the ADAF/RIAF branch, the disk temperature is independent of the mass accretion rate and is nearly constant at $T_{0} \sim 10^{11} \mathrm{~K}$. In contrast, on the optically thin low- $\beta$ branches, the disk temperature anti-correlates with the mass accretion rate, $\dot{M} \propto T_{0}^{-1 / 2}$, and $T_{0} \sim 10^{7-11} \mathrm{~K}$. However, it is not possible to compare these results directly with the observational data (e.g., the cutoff energy or the electron temperature in Mivakawa et al. 2008) because the disk temperature does not represent the electron temperature. We will discuss this issue in $\S 4.2$.

Figure 4 shows the same thermal equilibrium curves as in figure 2 but for $\alpha=0.01$. Compared to the results for $\alpha=0.05$, the ADAF/RIAF solution disappears at the lower mass accretion rate $\left(\dot{M}_{\mathrm{c}, \mathrm{A}} \sim 0.003 \dot{M}_{\mathrm{Edd}}\right)$.

\subsection{The Radial Structure of the Disks at Thermal Equilibrium}

In the previous subsection, we obtained local thermal equilibrium curves by solving the basic equations for various $\dot{M}$ and fixed parameters, $\alpha$, $\zeta$, and $\varpi$. In this subsection, we study the dependence of the solutions on $\varpi$ by fixing the parameters, $\alpha, \zeta$, and $\dot{M}$. Since we focus on the hardto-soft transition of the BHCs, we assume that a disk is in the ADAF/RIAF state corresponding to the low/hard state when the mass accretion rate is low. Thus we choose the ADAF/RIAF solution when three solutions (ADAF/RIAF, SLE, and low- $\beta$ or standard disk solution) are found for the same mass accretion rate.

Figure 5 shows the radial structure of a disk for $\alpha=0.05, \quad \zeta=0.6, \dot{M} / \dot{M}_{\text {Edd }}=$ $8.28 \times 10^{-3}$ (thick solid), 0.05 (dashed), 0.10 (dotdashed), 0.13(dotted), 0.45 (triple-dot-dashed), and 12.0 (thin solid), respectively.

When the mass accretion rate is low $(\dot{M} \lesssim$ $\left.0.01 \dot{M}_{\text {Edd }}\right)$, the dissipated energy is advected inward because radiative cooling is inefficient due to the low surface density over the whole disk. The whole disk is hot and the gas pressure is dominant, that is, the disk is in the ADAF/RIAF state.

As the mass accretion rate increases $\left(0.01 \dot{M}_{\text {Edd }} \lesssim\right.$ $\dot{M} \lesssim 0.2 \dot{M}_{\text {Edd }}$, the disk undergoes a transition to 
the optically thin low- $\beta$ disk from the outer radii because the mass accretion rate exceeds $\dot{M}_{\mathrm{c}, \mathrm{A}}$ at the transition radius. Now let us derive the radial dependence of $\dot{M}_{\mathrm{c}, \mathrm{A}}$. We find from the local thermal equilibrium curves that the ADAF/RIAF branch in which the heating rate balances the heat advection rate $\left(Q^{+} \sim Q_{\text {adv }}\right)$ intersects with the SLE branch in which the heating rate balances the optically thin radiative cooling rate $\left(Q^{+} \sim Q_{\text {thin }}^{-}\right)$at $\dot{M}_{\text {c,A. }}$. The gas pressure is dominant in both branches $\left(W_{\text {tot }} \sim W_{\text {gas }}\right)$. Using these relations, we find from the basic equations that $\dot{M}_{\mathrm{c}, \mathrm{A}} \propto \alpha^{2} \varpi^{5} \Omega_{\mathrm{K} 0}^{-1}\left|d \Omega_{\mathrm{K} 0} / d \varpi\right|^{3}\left(\ell_{\mathrm{K} 0}-\ell_{\mathrm{in}}\right)$. Since $\dot{M}_{\text {c, A }}$ increases inward in the outer region, the transitions take place from the outer radius. We note that $\dot{M}_{\mathrm{c}, \mathrm{A}}$ has its maximum value around $10 r_{\mathrm{s}}$ for $\ell_{\mathrm{in}}=\ell_{\mathrm{K} 0}\left(3 r_{\mathrm{s}}\right)$. Therefore, the innermost region $\left(\varpi \lesssim 10 r_{\mathrm{s}}\right)$ undergoes a transition to the optically thin low- $\beta$ disk at a lower mass accretion rate than in the slightly further out region $\left(10 r_{\mathrm{s}} \lesssim \varpi \lesssim 30 r_{\mathrm{s}}\right)$. If we consider transonic solutions for which $\ell_{\text {in }}<\ell_{\mathrm{K} 0}$, such a feature may not appear.

When $0.2 \dot{M}_{\text {Edd }} \lesssim \dot{M} \lesssim 5 \dot{M}_{\text {Edd }}$, the whole disk becomes an optically thin low- $\beta$ disk in which the dissipated energy is radiated by the bremsstrahlung emission. We note that when $\zeta=0.6$, an optically thick low- $\beta$ disk does not appear because the large magnetic flux prevents the disk from shrinking in the vertical direction. Let us derive the radial dependence of the surface density and the temperature in optically thin low- $\beta$ disks. In an optically thin low- $\beta$ disk, the magnetic pressure is dominant ( $W_{\text {tot }} \sim W_{\text {mag }}$ ) and the magnetic heating rate balances the optically thin radiative cooling rate $\left(Q^{+} \sim Q_{\text {thin }}^{-}\right)$. Using these relations, we find from the basic equations that $\Sigma \propto \dot{M} \alpha^{-1} \varpi^{4 \zeta / 5-2}\left(\ell_{\mathrm{K} 0}-\ell_{\mathrm{in}}\right)^{3 / 5} \Omega_{\mathrm{K} 0}^{-2 / 5}$ and $T_{0} \propto \dot{M}^{-2} \alpha^{4} \varpi^{6-4 \zeta}\left(d \Omega_{\mathrm{K} 0} / d \varpi\right)^{2}$. In the outer region where $\Omega_{\mathrm{K} 0} \propto \varpi^{-3 / 2}$ and $\left(\ell_{\mathrm{K} 0}-\ell_{\mathrm{in}}\right) \sim \ell_{\mathrm{K} 0} \propto$ $\varpi^{1 / 2}$, we find that $\Sigma \propto \dot{M} \alpha^{-1} \varpi^{4 \zeta / 5-11 / 10}$ and $T_{0} \propto \dot{M}^{-2} \alpha^{4} \varpi^{1-4 \zeta}$.

When the mass accretion rate is high $(\dot{M} \gtrsim$ $5 \dot{M}_{\text {Edd }}$ ), the disk undergoes a transition to a slim disk in the inner region because the radial heat advection becomes efficient again. We note that even in a slim disk the magnetic pressure is comparable to or larger than the radiation pressure $\left(W_{\text {mag }} \gtrsim W_{\text {rad }} \gg W_{\text {gas }}\right)$ for $\zeta=0.6$.
Figure 6] shows the results for $\zeta=0$. As the mass accretion rate increases $\left(0.01 \dot{M}_{\text {Edd }} \lesssim \dot{M} \lesssim\right.$ $\left.0.2 \dot{M}_{\text {Edd }}\right)$, the disk undergoes a transition to a low- $\beta$ disk from the outer radii, and the whole disk becomes a low- $\beta$ disk when $0.2 \dot{M}_{\text {Edd }} \lesssim \dot{M} \lesssim$ $5 \dot{M}_{\text {Edd }}$. The low- $\beta$ disks consist of two parts, an inner optically thick part and an outer optically thin part. In an optically thick low- $\beta$ disk, since $Q^{-} \sim Q_{\text {thick }}^{-} \propto 16 \sigma I_{N} T_{0}^{4} /(3 \tau / 2) \propto T_{\text {eff }}^{4}$, we obtain the radial dependence of the effective temperature, $T_{\text {eff }} \propto \varpi^{-3 / 4}$. This dependence is the same as that in the standard disk. In addition, when the mass accretion rate is high $\left(\dot{M} \gtrsim 5 \dot{M}_{\text {Edd }}\right)$, slim disks consist of an inner radiation pressure dominated region and an outer magnetic pressure dominated region.

An example of an extremely weak magnetic field model $(\zeta=-1.8)$ is shown in figure 7 . In this model, the inner region of a disk undergoes a transition to a radiation pressure dominated standard disk while the outer region undergoes a transition to an optically thick low- $\beta$ disk. As a result, the inner region of a disk always stays in the high $\beta$ state.

When $\alpha=0.05$, the gas pressure dominated standard disk solutions do not appear because $\dot{M}_{\text {c,A }}$, being proportional to $\alpha^{2}$, is higher than $\dot{M}_{\mathrm{c}, \mathrm{S}}$ for $\alpha=0.05$. The transition to the gas pressure dominated standard disk takes place when $\alpha$ is sufficiently small so that $\dot{M}_{\mathrm{c}, \mathrm{A}}<\dot{M}_{\mathrm{c}, \mathrm{S}}$. In figure 8, we show the results for $\alpha=0.01$, $\zeta=-1.8, \dot{M} / \dot{M}_{\text {Edd }}=1 \times 10^{-3}$ (thick solid), $2.02 \times 10^{-3}$ (dashed), $4.09 \times 10^{-3}$ (dot-dashed), $2.68 \times 10^{-2}$ (dotted), 1.15(triple-dot-dashed), and 12.0 (thin solid), respectively. As the mass accretion rate increases $\left(1 \times 10^{-3} \dot{M}_{\mathrm{Edd}} \lesssim \dot{M} \lesssim\right.$ $\left.0.03 \dot{M}_{\text {Edd }}\right)$, the disk undergoes a transition to a gas pressure dominated standard disk in the inner region while an optically thick low- $\beta$ disk exists in the outer region.

\section{Discussion}

\subsection{New Thermal Equilibrium Solution connecting an Optically Thin Regime and an Optically Thick Regime}

We obtained thermal equilibrium curves for optically thin and optically thick accretion disks incorporating the mean azimuthal magnetic fields. We prescribed that the azimuthally averaged 
Maxwell stress is proportional to the total (gas, radiation, and magnetic) pressure. Consequently, the heating rate can be large if the magnetic pressure is high even when the gas pressure and the radiation pressure are low. To complete the set of basic equations, we specified the magnetic flux advection rate rather than the plasma $\beta$ at each radius. Hence, a decrease in temperature via the cooling instability results in an increase in magnetic pressure due to the conservation of the azimuthal magnetic flux $\left\langle B_{\varphi}\right\rangle H$.

Under these assumptions, we obtained low- $\beta$ solutions in both the optically thin regime and the optically thick regime in addition to the ADAF/RIAF, SLE, standard, and slim disk solutions. In the low- $\beta$ disks, the magnetic heating balances the radiative cooling. The slopes of the thermal equilibrium curves plotted in the $\Sigma$ vs. $T$ plane in figure 2 and figure 4 indicate that the low$\beta$ disk is thermally stable. The borders between the optically thin and thick parts of the low- $\beta$ branches depend on $\zeta$ which specifies the magnetic flux at each radius. As the magnetic flux advection rate increases, the magnetic pressure becomes higher, therefore the magnetic heating rate increases. Hence the equilibrium temperature becomes higher, and the surface density decreases. Thus, the effective optical depth decreases for the same mass accretion rate. This means that the optically thin part of the low- $\beta$ branch extends to a higher mass accretion rate as $\zeta$ increases.

We also studied the radial dependence of the solutions. The ADAF/RIAF undergoes a transition to the low- $\beta$ disk from the outer radii as the mass accretion rate increases. This is because $\dot{M}_{\text {c, A }}$ at outer radii is lower than its value at inner radii. Figure 9 shows schematic pictures of configurations of accretion disks for various $\dot{M}$ and $\zeta$.

Let us discuss the stability of low- $\beta$ disks. Shibata, Tajima, \& Matsumoto (1990) carried out MHD simulations of the buoyant escape of the magnetic flux due to the Parker instability. They showed that once a disk is dominated by the magnetic pressure, it can stay in the low- $\beta$ state because the strong magnetic tension suppresses the growth of the Parker instability. Mineshige et al. (1995) suggested that such low- $\beta$ disks emit hard X-rays. When $\beta \sim 1$ in the surface of the low$\beta$ disk, the buoyant escape of the magnetic flux from the surface layer will reduce the total azimuthal magnetic flux of the disk. This loss of magnetic flux can balance the amplification of magnetic fields due to the MRI in this layer. The results of three-dimensional global MHD simulations by Machida et al. (2006) indicate that low- $\beta$ disks stay in a quasi-equilibrium state at least for a thermal time scale. The longer time scale evolution of the low- $\beta$ disk is a subject to be studied in near future.

\subsection{Bright Hard State undergoing Bright/Slow Transition}

One of the main aims of this paper is to explain the bright hard state observed in rising phases of the outbursts of BHCs (e.g., Miyakawa et al. 2008). Figure 3 shows that the optically thin part of the low- $\beta$ branch extends to $\gtrsim 0.1 \dot{M}_{\mathrm{Edd}}$ for $\zeta \gtrsim 0.3$. In the ADAF/RIAF branches, the disk temperature is independent of the mass accretion rate and remains nearly at the virial temperature. This is consistent with the fact that the cutoff energy observed in the low/hard state of BHCs is independent of their luminosity. In contrast, the disk temperature roughly anti-correlates with the mass accretion rate in the optically thin low- $\beta$ branches. This indicates that the electron temperature in the hard X-ray emitting optically thin low- $\beta$ disks anti-correlates with the luminosity. This feature is consistent with the anti-correlation observed in the bright hard state of BHCs.

In addition, the optically thin low- $\beta$ disks explain the bright/slow transition reported by Gierliński \& Newton (2006). For small $\zeta$, the ADAF/RIAF directly undergoes a transition to the optically thick disks emitting the soft X-rays at $\sim 0.1 \dot{M}_{\text {Edd }}$ for $\alpha=0.05$ (panel $(\mathrm{h}) \rightarrow(\mathrm{g}) \rightarrow$ (f) in figure 9). This corresponds to the dark/fast transition (see Gierliński \& Newton 2006). In contrast, for large $\zeta$, the ADAF/RIAF first undergoes a transition to the optically thin low- $\beta$ disk, which can be identified as the bright hard state. Subsequently, this disk undergoes a transition to the optically thick disk at a mass accretion rate higher than the rate for small $\zeta$. This transition corresponds to the bright/slow transition. (panel (h) $\rightarrow(\mathrm{j}) \rightarrow(\mathrm{i}) \rightarrow(\mathrm{e})$ in figure 9)

It is anticipated that if the magnetic flux escapes from the disk due to magnetic buoyancy or dissipates due to magnetic reconnection, the equi- 
librium curve may shift to the one for smaller $\zeta$. In such a case, the optically thin low- $\beta$ disk immediately undergoes a transition to the standard disk or the optically thick low- $\beta$ disk. This transition may accompany the ejection of jets observed during the hard-to-soft transition of BHCs because magnetic energy stored in the low- $\beta$ disk is released.

Although we have adopted the single temperature model $\left(T_{\mathrm{i}}=T_{\mathrm{e}}\right)$, it has been pointed out that the electron temperature becomes lower than the ion temperature $\left(T_{\mathrm{i}} \sim 10^{11-12} \mathrm{~K}\right)$ in such a low density, high temperature region (e.g., Nakamura et al. 1996, 1997). Moreover, cooling via the synchrotron emission and the inverseCompton effect can be efficient. By contrast, the electron temperature and the ion temperature coincide $\left(T_{\mathrm{i}} \sim T_{\mathrm{e}} \sim 10^{6-7} \mathrm{~K}\right)$ in low temperature and high density disks such as optically thick low- $\beta$ disks, standard disks, and slim disks. In subsequent papers, we will take into account the decoupling of ions and electrons in the low density, high temperature region and incorporate the synchrotron emission and the inverse-Compton effect, in addition to the bremsstrahlung emission as the cooling mechanism.

\subsection{Magnetically Supported Thermal Disks}

We obtained solutions for the optically thick low- $\beta$ disks emitting blackbody radiation (panel (f) in figure 9). The radial distribution of the effective temperature in the optically thick low- $\beta$ disk is roughly the same as that for the standard disk $\left(T_{\text {eff }} \propto \varpi^{-3 / 4}\right)$ because it does not depend on whether the gas pressure or the magnetic pressure is dominant as long as the heating rate balances the blackbody cooling. This indicates that the optically thick low- $\beta$ disk will be observed as a thermal disk.

The seed photons incident on the Compton corona (the low density, high temperature region, that is, the ADAF/RIAF or the optically thin low$\beta$ region) have been thought to be provided by a cool disk or by synchrotron emissions. The optically thick low- $\beta$ disk can also be the source of seed photons. When $\zeta \lesssim 0$, the ADAF/RIAF undergoes a transition to the optically thick low- $\beta$ disk from the radially outer region as the mass accretion rate increases. Thus, the inner ADAF/RIAF region is surrounded by the outer optically thick low- $\beta$ disk (panel (g) in figure 9). An increase in seed photons can result in a decrease in the temperature of the Compton corona. Furthermore, the hard X-rays Comptonized in the corona can be incident on the optically thick low- $\beta$ disk and can be partly reflected. As a result, the existence of such region will enhance the Compton continua with a reflection component in the X-ray spectrum.

To sum up, the optically thick low- $\beta$ disk not only provides seed photons to the Compton corona but also produces a soft spectral excess and a reflection component observed in the low/hard state of Cyg X-1 (Makishima et al. 2008) and GRO J1655-40 (Takahashi et al. 2008) at the Suzaku Xray observatory.

\subsection{Limit Cycle Oscillation Between an Optically Thick Low- $\beta$ Disk and a Slim Disk}

So far, we have focused on the hard-to-soft transition of BHCs. Now we discuss X-ray flares as observed in GRS 1915+105 (e.g., Belloni et al. 1997; Taam, Chen, \& Swank 1997; Paul et al. 1998; Belloni et al. 2000). This object frequently exhibits state transitions and quasi-regular Xray flares. It has been thought that the latter effect may be driven by limit cycle oscillations between the standard disk and the slim disk (panel (a) $\leftrightarrow$ (b) in figure 9). Ohsuga (2006, 2007) reproduced such limit cycle evolution by global two-dimensional radiation hydrodynamic (RHD) simulations of optically thick accretion disks using the flux-limited diffusion (FLD; Levermore \& Pomraning 1981) approximation for the radiation transfer and the phenomenological $\alpha$ viscosity for the angular momentum transfer and the viscous heating. However, such RHD simulations do not follow the formation of the low- $\beta$ disk presented in this paper.

We first consider a transition from a slim disk to an optically thick low- $\beta$ disk. We suppose that the disk is initially in the slim disk state in which magnetic heating balances the heat advection (the photon trapping) and that these are greater than the radiative cooling $\left(Q^{+} \sim Q_{\mathrm{adv}}>Q^{-}\right)$. Figure 2 shows that as the mass accretion rate decreases, the slim disk branches disappear. At this point, the magnetic heating (and the heat advection) become less than the radiative cooling 
$\left(Q^{+} \sim Q_{\mathrm{adv}}<Q^{-}\right)$. Therefore cooling instability occurs. As a result, the disk shrinks in the vertical direction along the cooling timescale and undergoes a transition to an optically thick low- $\beta$ disk (panel (e) $\rightarrow$ (f) in figure 9). This situation is similar to the transition from the ADAF/RIAF to the optically thin low- $\beta$ disk found by Machida et al. (2006). Subsequently, as the mass accretion rate increases, the disk undergoes a transition back to a slim disk because the optically thick low- $\beta$ branches disappear (panel (f) $\rightarrow$ (e) in figure 9).

We emphasize that an optically thick low- $\beta$ disk undergoes a transition to a slim disk at higher mass accretion rates $\left(\sim \dot{M}_{\text {Edd }}\right.$ for $\alpha=0.5$ and $\zeta=0)$ than those at which a standard disk undergoes a transition to a slim disk $\left(\sim 0.1 \dot{M}_{\text {Edd }}\right)$. This results in a small variation in luminosity for quasi-regular X-ray flares compared to that expected from the limit cycle between a standard disk and a slim disk.

\section{Summary}

We have obtained low- $\beta$ solutions for optically thin and optically thick disks incorporating the mean azimuthal magnetic fields. The key assumptions are (1) we prescribed the Maxwell stress to be proportional to the total (gas, radiation, and magnetic) pressure, and (2) we specified the magnetic flux advection rate instead of the plasma $\beta$ at each radius. As a result, the strong magnetic fields mainly contribute to the heating and increase the half thickness of the disk. In the low- $\beta$ disks, the heating enhanced by strong magnetic pressure balances the radiative cooling rather than the heat advection. Optically thin low- $\beta$ disks explain the bright hard state undergoing the bright/slow transition observed in BHCs. The optically thick low$\beta$ disk can be observed as a thermal disk, which can supply seed photons into the Compton corona (the ADAF/RIAF or the optically thin low- $\beta$ region) and reflect incident photons from the Compton corona. We pointed out the possibility of new limit cycle oscillations between an optically thick low- $\beta$ disk and a slim disk, which will exhibit a smaller variation in luminosity than that expected from a limit cycle between a standard disk and a slim disk.

We are grateful to T. Hanawa, S. Mineshige for discussion. We also acknowledge T. Miyakawa for valuable comments and discussions from observational points of view, and B. Prager for helpful comments on the manuscript. This work is supported by the Grant-in-Aid for Scientific Research of the Ministry of Education, Culture, Sports, Science and Technology (RM: 20340040) and Research Fellowships of Japan Society for the Promotion of Science for Young Scientists. 


\section{REFERENCES}

Abramowicz, M. A., Chen, X. M., Granath, M., \& Lasota, J. P. 1996, ApJ, 471, 762

Abramowicz, M. A., Chen, X., Kato, S., Lasota, J. P., \& Regev, O. 1995, ApJ, 438, L37

Abramowicz, M. A., Czerny, B., Lasota, J. P., \& Szuszkiewicz, E. 1988, ApJ, 332, 646

Balbus, S. A. \& Hawley, J. F. 1991, ApJ, 376, 214

Belloni, T., Klein-Wolt, M., Meńdez, M., van der Klis, M. \& van Paradijs, J. 2000, A\&A, 355, 271

Belloni, T., Meńdez, M., King, A. R., van der Klis, M. \& van Paradijs, J. 1997, ApJ, 479, L145

Done, C. \& Gierliński, M. 2003, MNRAS, 342, 1041

Eardley, D. M., Lightman, A. P., \& Shapiro, S. L. 1975, ApJ, 199, L153

Esin, A., McClintock, J. E., Narayan, R. 1997, ApJ, 489, 865

Gierliński, M. \& Newton, J. 2006, MNRAS, 370, 837

Hawley, J. F. 2000, ApJ, 528, 462

Hawley, J. F. \& Krolik, J. H. 2001, ApJ, 548, 348

Hawley, J. F. \& Krolik, J. H. 2002, ApJ, 566, 164

Hirose, S., Krolik, J. H., \& Stone, J. M. 2006, ApJ, 640, 901

Hubeny, I. 1990, ApJ, 351, 632

Ichimaru, S. 1977, ApJ, 214, 840

Kato, S., Fukue, J., \& Mineshige, S. 2008, Black-Hole Accretion Disks: Towards a New Paradigm (Kyoto: Kyoto University Press)

Kawaguchi, T. 2003, ApJ, 593, 69

Komissarov, S. S. 2006, MNRAS, 368, 993

Krolik, J. H., Hirose, S., \& Blaes, O. 2007, ApJ, 664, 1045

Levermore, C. D. \& Pomraning, G. C. 1981, ApJ, 248,321
Machida, M., Hayashi, M. R., \& Matsumoto, R. 2000, ApJ, 532, L67

Machida, M. \& Matsumoto, R. 2003, ApJ, 585, 429

Machida, M., Nakamura, K. E., \& Matsumoto, R. 2004, PASJ, 56, 671

Machida, M., Nakamura, K. E., \& Matsumoto, R. 2006, PASJ, 58, 193

Makishima, K., Takahashi, H., Yamada, S. et al. 2008, PASJ, 60, 585

Mineshige, S., Kusunose, M. \& Matsumoto, R. 1995, ApJ, 445, L43

Miyakawa, T., Yamaoka, K., Homan, J., Saito, K., Dotani, T., Yoshida, A., \& Inoue, H 2008, PASJ, 60, 637

Nakamura, K. E., Matsumoto, R., Kusunose, M., \& Kato, S. 1996, PASJ, 48, 761

Nakamura, K. E., Kusunose, M., Matsumoto, R., \& Kato, S. 1997, PASJ, 49, 503

Narayan, R. \& Yi, I. 1994, ApJ, 428, L13

Narayan, R. \& Yi, I. 1995, ApJ, 452, 710

Nishikori, H, Machida, M, \& Matsumoto, R. ApJ, 2006, 641, 862

Oda, H., Machida, M., Nakamura, K. E., \& Matsumoto, R. 2007, PASJ, 59, 457

Ohsuga, K. 2006, ApJ, 640, 923

Ohsuga, K. 2007, ApJ, 659, 205

Okada, R., Fukue, J. \& Matsumoto, R. 1986, PASJ, 41, 133

Paczyńsky, B. \& Wiita, P. J. 1980, A\&A, 88, 23

Parker, E. N. 1966, ApJ, 145, 811

Paul, B., Agrawal, P. C., Rao, A. R., Vahia, M. N., \& Yadav, J. S. 1998, ApJ, 492, L63

Shakura, N. I. \& Sunyaev, R. A. 1973, A\&A, 24, 337

Shapiro, S. L., Lightman, A. P., \& Eardley, D. M. 1976, ApJ, 204, 187 
Shibata, K., Tajima, T., \& Matsumoto, R. 1990, ApJ, 350, 295

Shibazaki, N. \& Hōshi, R. 1975, Progress of Theoretical Physics, 54, 706

Taam, R. E., Chen, X., \& Swank, J. H. 1997, ApJ, 485, L83

Takahashi, H., Fukazawa, Y., Mizuno, T et al. 2008, PASJ, 60, S69

Thorne, K. S. \& Price, R. H. 1975, ApJ, 195, L101

Vierdayanti, K., Mineshige, S., Ebisawa, K, \& Kawaguchi, T. 2006, PASJ, 58, 915

Vierdayanti, K., Watarai, K, \& Mineshige, S. 2008, PASJ, 60, 653

This 2-column preprint was prepared with the AAS IATEX macros v5.2. 
(a)

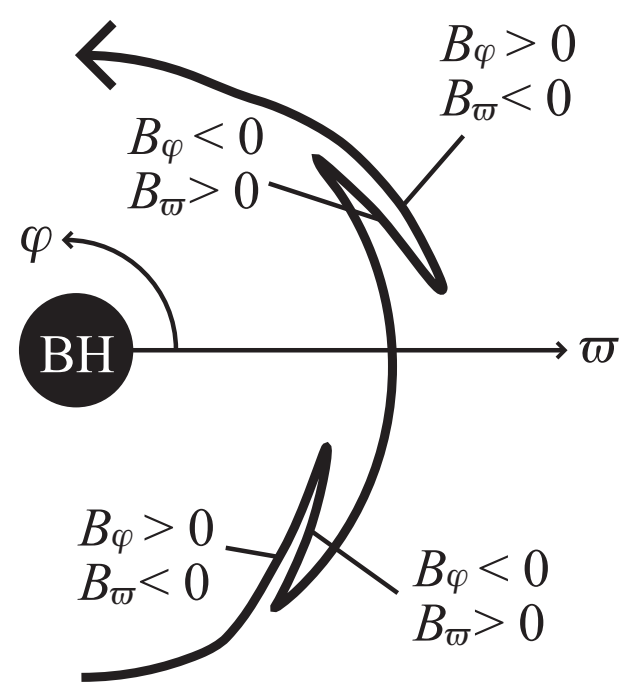

(b)
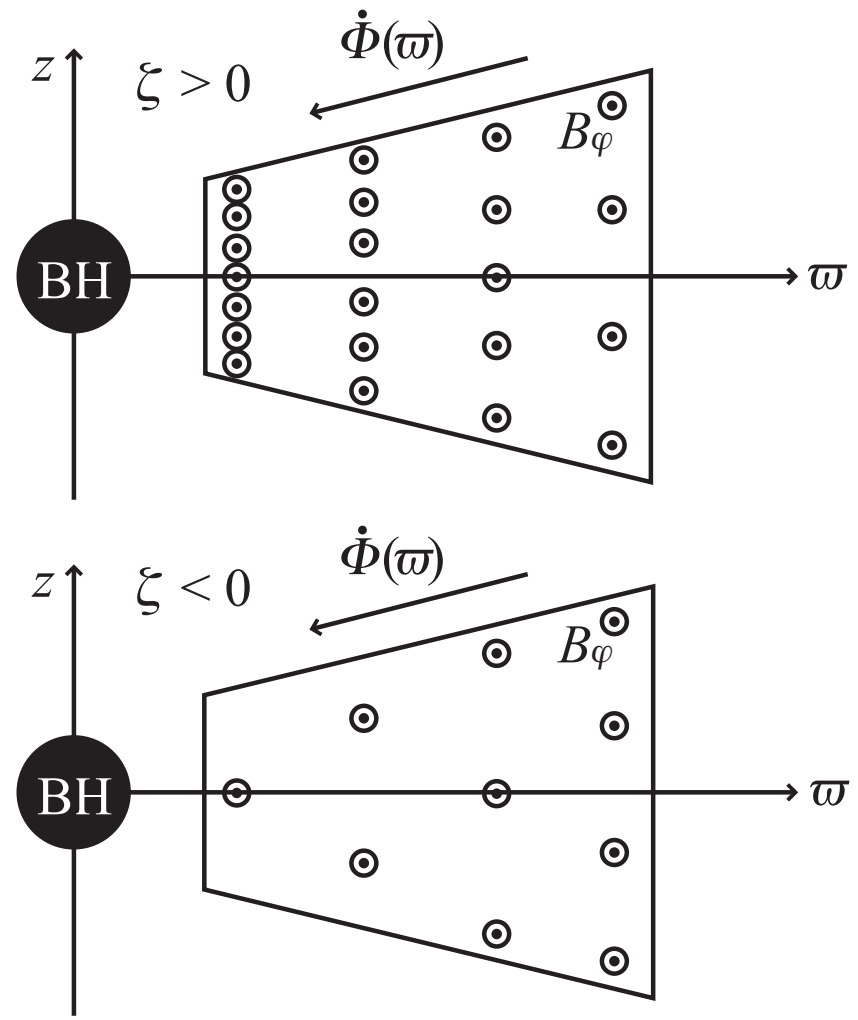

Fig. 1.- Schematic picture of magnetic field lines inside the accretion disk. (a) The magnetic fields are decomposed into the mean azimuthal magnetic fields and fluctuating fields. The azimuthal average of the radial component of the magnetic fields, $\left\langle B_{\varpi}\right\rangle$, is small because positive and negative $B_{\varpi}$ cancel out. However, the azimuthal average of the product of the radial and azimuthal component $\left\langle B_{\varpi} B_{\varphi}\right\rangle$ has a large negative value because $B_{\varpi} B_{\varphi}$ does not change sign when magnetic fields are deformed by nonlinear growth of the MRI. (b) The advection of the azimuthal magnetic flux. The radial dependence of the magnetic flux advection rate $\dot{\Phi}$ is parameterized by $\zeta$. When $\zeta>0, \dot{\Phi}$ increases with a decreasing radius. However, $\dot{\Phi}$ decreases when $\zeta<0$. The mean azimuthal magnetic flux is conserved in the radial direction when $\zeta=0$. 


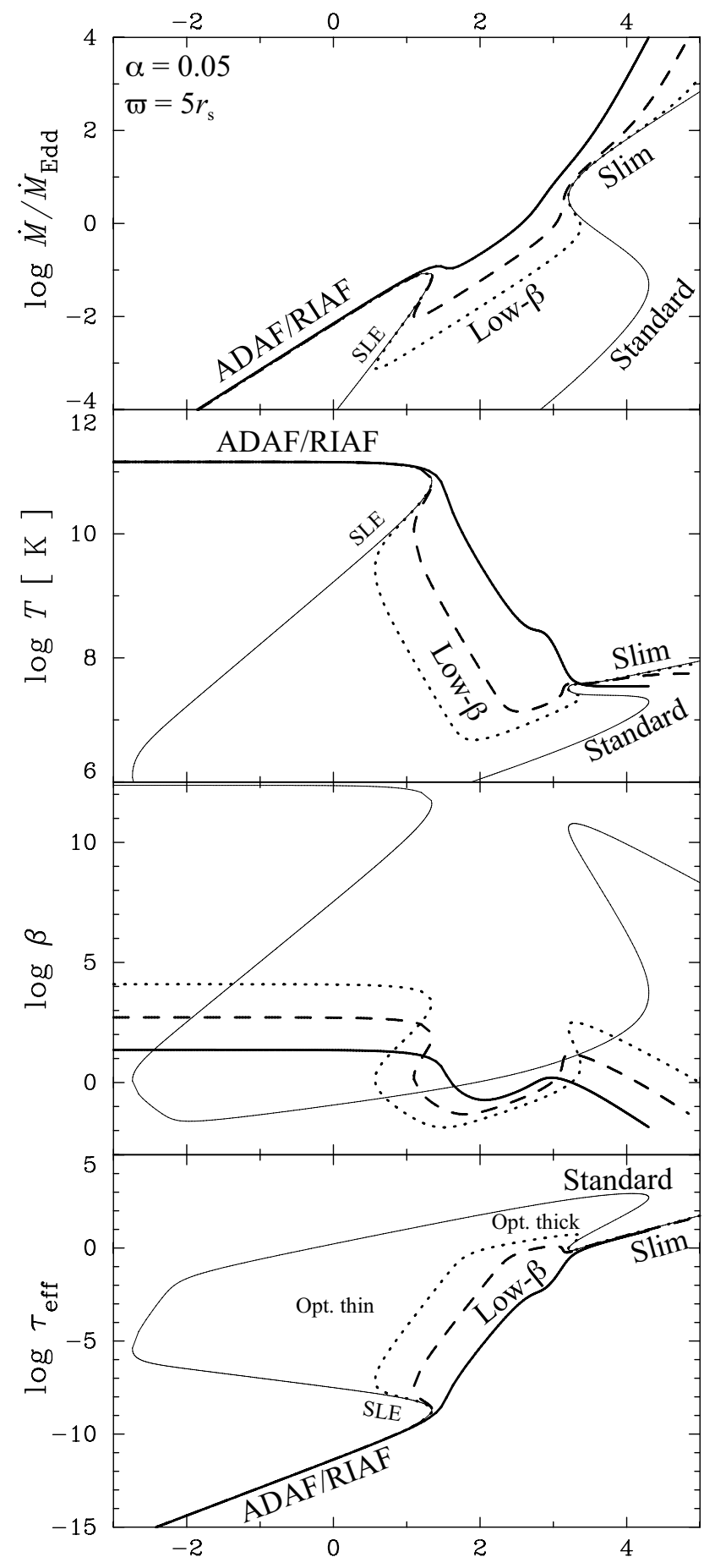

$\log \Sigma\left[\mathrm{g} / \mathrm{cm}^{2}\right]$

Fig. 2.- Local thermal equilibrium curves of accretion disks at $\varpi=5 r_{\mathrm{s}}$ on the $\Sigma$ vs. $\dot{M} / \dot{M}_{\mathrm{Edd}}, T, \beta$ and $\tau_{\text {eff }}$ plane for $\alpha=0.05, \zeta=0.6$ (thick solid), 0.3(dashed), 0 (dotted), and -1.8 (thin solid, extremely weak magnetic field case). $\dot{M}_{\mathrm{Edd}}=L_{\mathrm{Edd}} / \eta_{\mathrm{e}} c^{2}$ is the Eddington accretion rate, where the energy conversion efficiency is taken to be $\eta_{\mathrm{e}}=0.1$. The low- $\beta$ branches connect the optically thin and thick regimes. 


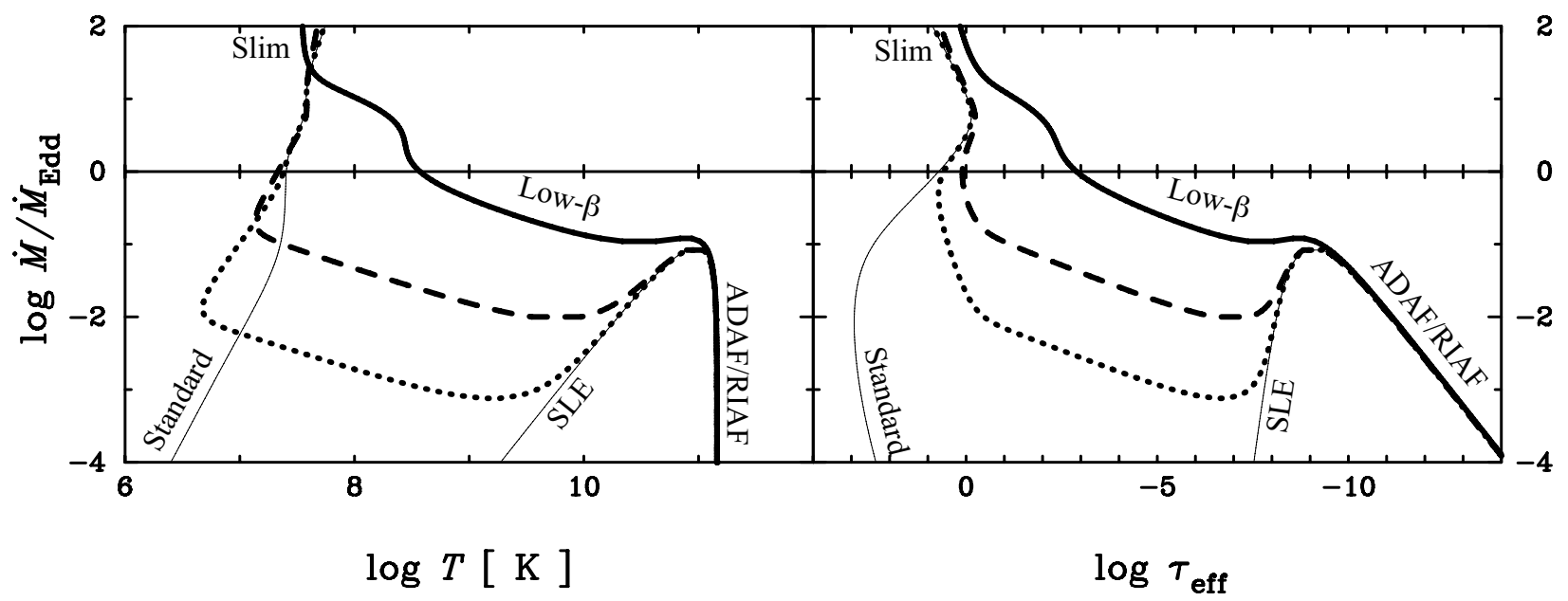

Fig. 3.- Same as figure 2 but plotted in the $T$ vs. $\dot{M} / \dot{M}_{\text {Edd }}$ plane and the $\tau_{\text {eff }}$ vs. $\dot{M} / \dot{M}_{\text {Edd }}$ plane. The temperature $T$ anti-correlates with $\dot{M}$ on the optically thin low- $\beta$ branches. 


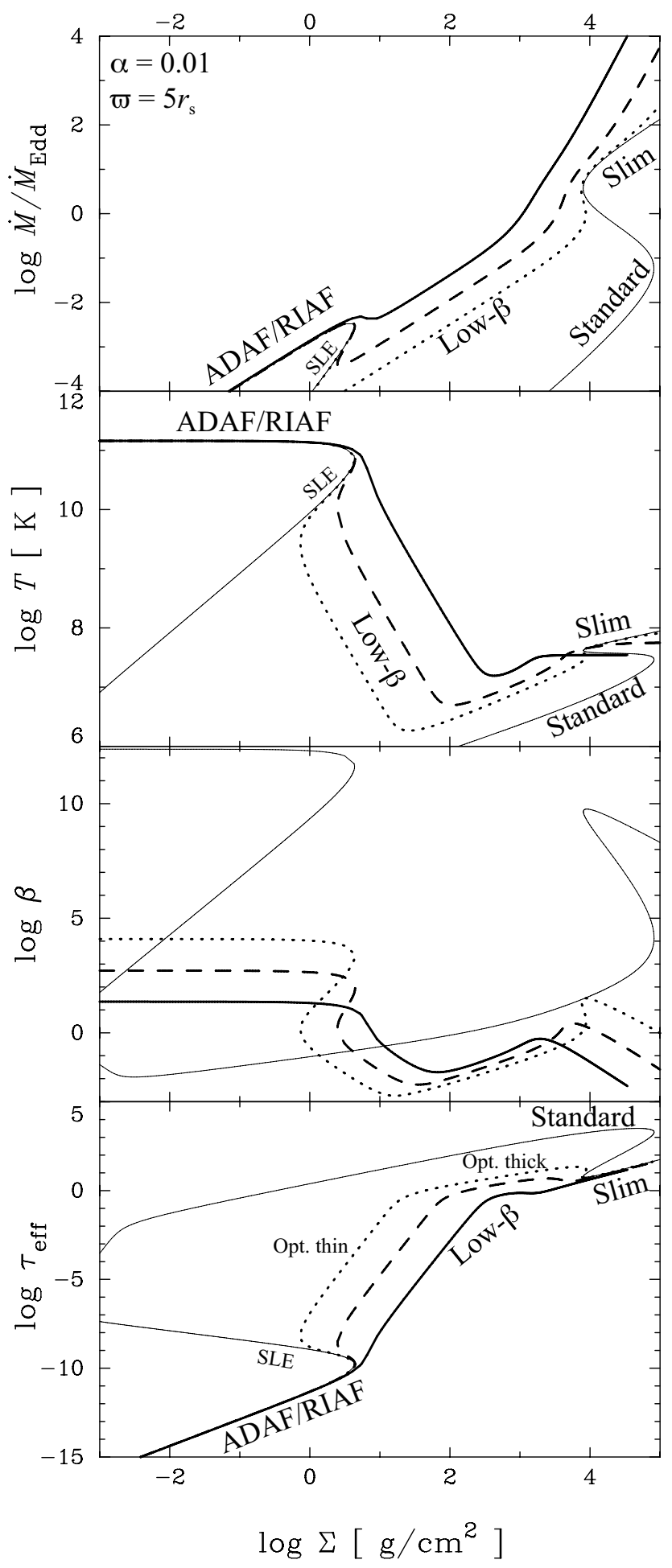

Fig. 4.- Same as figure 2, but for $\alpha=0.01$. 


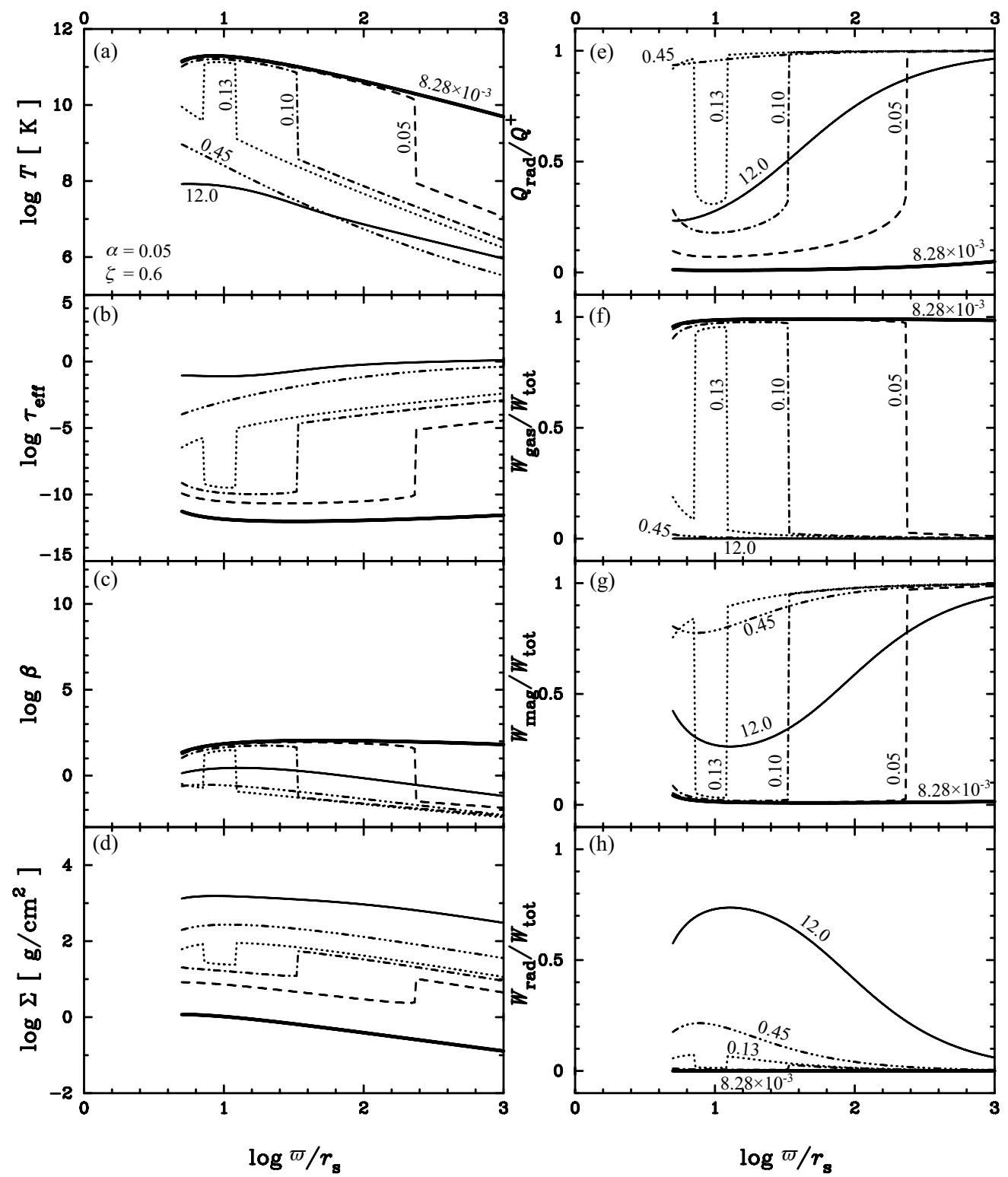

Fig. 5. - The radial dependence of (a), temperature $T$, (b) effective optical depth, $\tau_{\text {eff }},(\mathrm{c}) \beta=\left(p_{\text {gas }}+\right.$ $\left.p_{\text {rad }}\right) / p_{\text {mag }}$, (d) surface density $\Sigma$, (e) the ratio of the heating rate and the radiative cooling rate, the ratio of the vertically integrated total pressure and (f) the gas pressure $W_{\text {gas }}$, (g) the magnetic pressure $W_{\text {mag }}$, and (h) the radiation pressure $W_{\text {rad }}$ for $\alpha=0.05, \zeta=0.6, \dot{M} / \dot{M}_{\text {Edd }}=8.28 \times 10^{-3}$ (thick solid), 0.05 (dashed), 0.10 (dot-dashed), 0.13(dotted), 0.45(triple-dot-dashed), 12 (thin solid). We chose the ADAF/RIAF solution when we obtained three solutions (ADAF/RIAF, SLE and low- $\beta$ or standard disk solutions) for the same $\dot{M}$. 


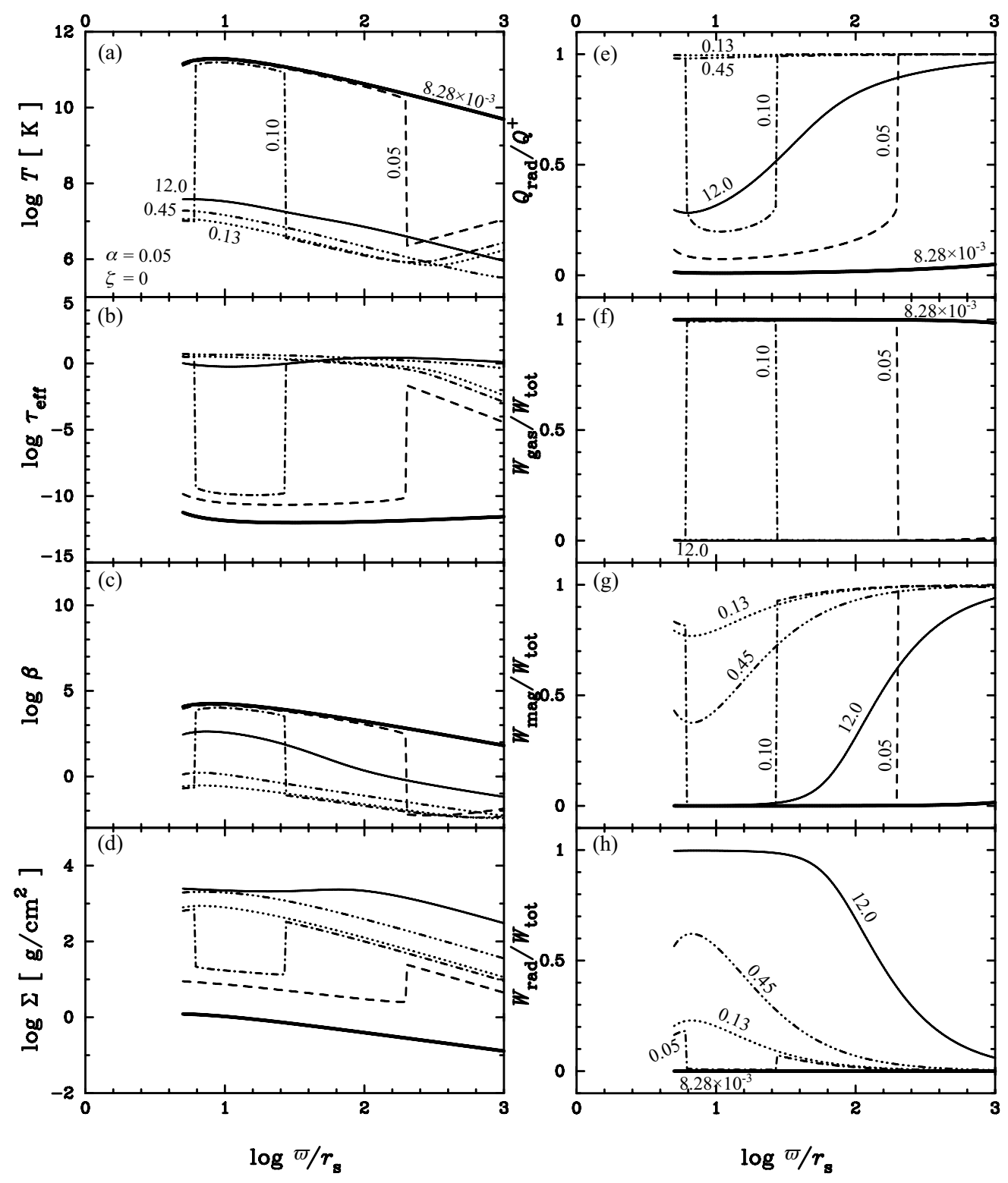

Fig. 6.- Same as figure 5, but for $\zeta=0$. 


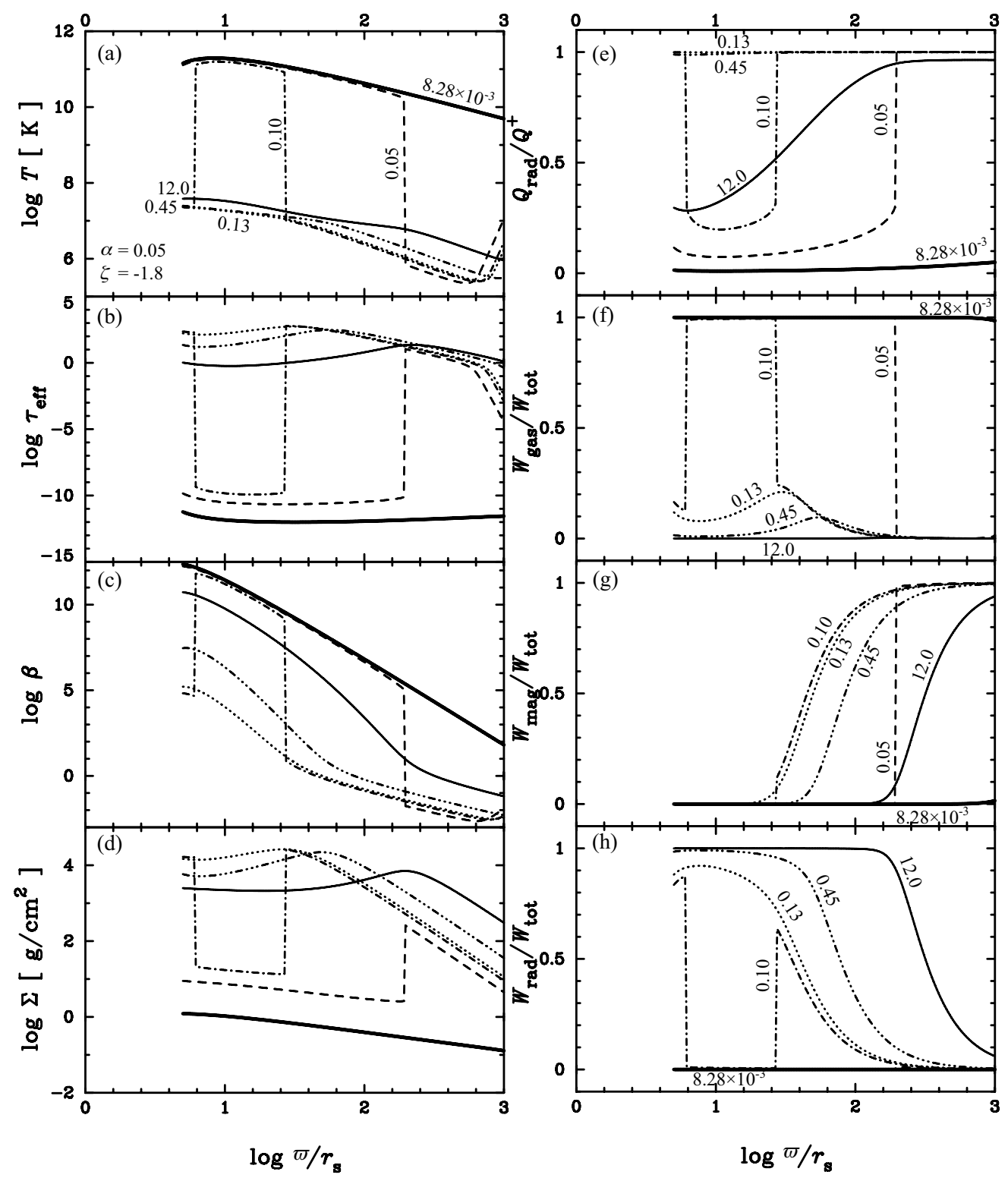

Fig. 7.- Same as figure 5, but for $\zeta=-1.8$. 


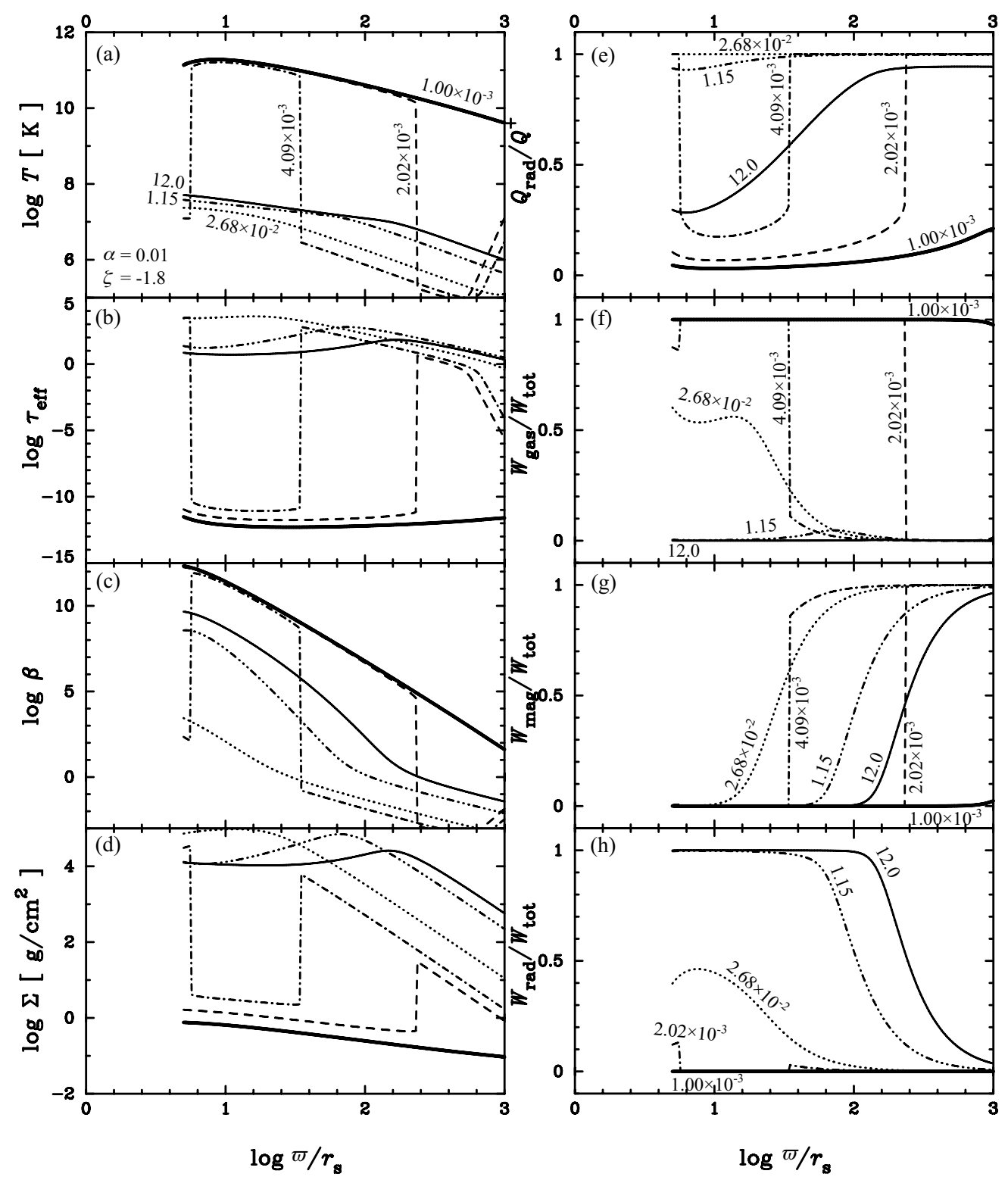

Fig. 8.- Same as figure [5, but for $\alpha=0.01, \zeta=-1.8, \dot{M} / \dot{M}_{\text {Edd }}=1.00 \times 10^{-3}$ (thick solid), $2.02 \times$ $10^{-3}$ (dashed), $4.09 \times 10^{-3}$ (dot-dashed), $2.68 \times 10^{-2}$ (dotted), 0.45 (triple-dot-dashed), 12 (thin solid). 

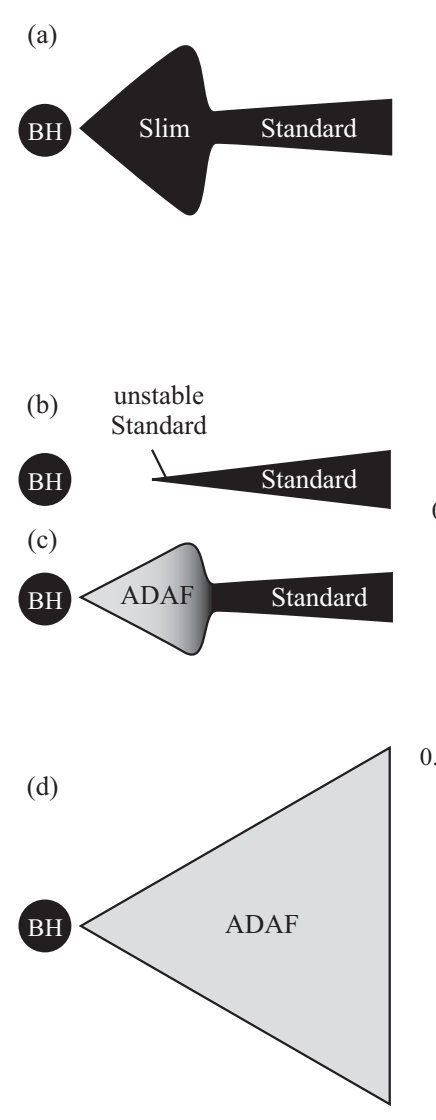

Conventional model (No Magnetic Field)

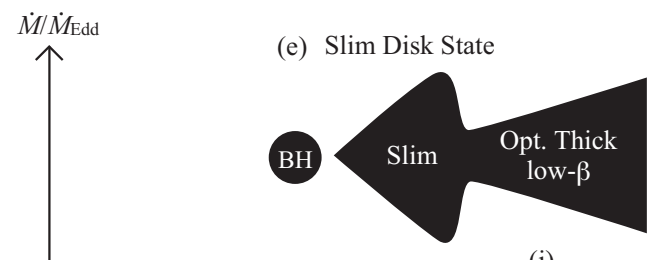

(i)

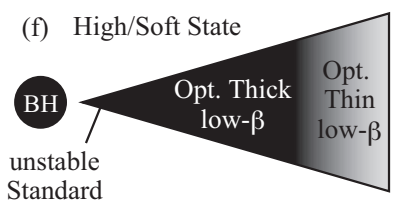

BH

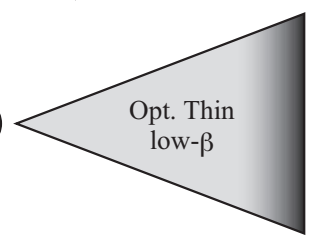

Bright Hard State

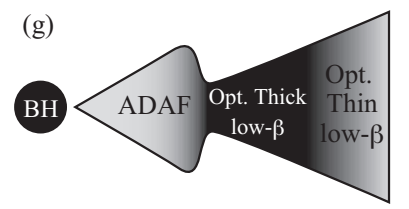

(j)

BH
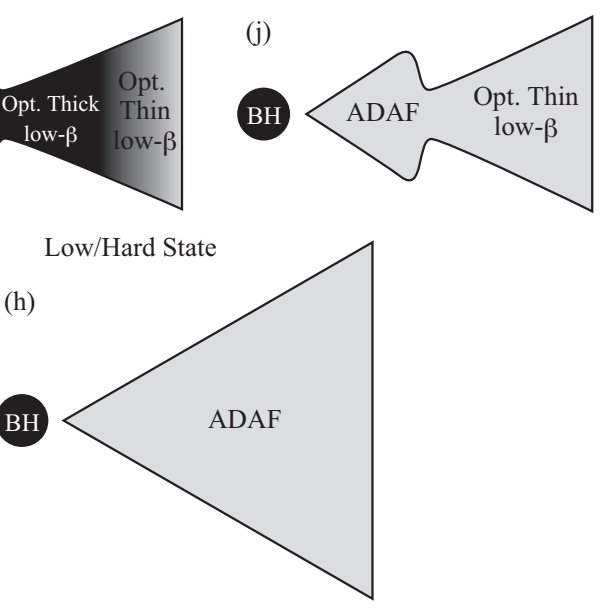

Small $\longleftarrow \xi$

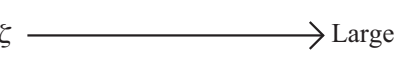

Fig. 9. - Schematic pictures of the configuration of accretion disks for various mass accretion rates and magnetic flux advection parameter $\zeta$. The optically thin region is indicated by gray, and the optically thick region is indicated by black. The panels $(\mathrm{c}),(\mathrm{d}),(\mathrm{g})$, and $(\mathrm{h})$ represent low mass accretion states corresponding to the low/hard state. The panels (i) and (j) represent mildly high mass accretion states for large $\zeta$ corresponding to the bright hard state. The panels (b) and (f) represent the mildly high mass accretion state for small $\zeta$ corresponding to the high/soft state. The slim disk state is illustrated in the top panels (a) and (e). The left column shows the configuration predicted by the conventional (no magnetic) model. 